\title{
BGP INTER-DOMAIN ROUTING TRAFFIC ANALYSIS
}

\author{
Huijun Yang \\ A thesis submitted to \\ the Faculty Graduate Studies and Research \\ in partial fulfillment of the requirements for the degree of \\ Master of Science \\ Information and Systems Science
}

Department of Systems and Computer Engineering

Carleton University

1125 Colonel By Drive

Ottawa, Ontario

K1S 5B6 Canada

(C) Huijun Yang

September, 2002 
National Library

of Canada

Acquisitions and Bibliographic Services

395 Wellington Street Ottawa ON K1A ON4 Canada
Bibliothèque nationale

du Canada

Acquisitions et services bibliographiques

395, rue Wellington

Ottawa ON K1A ON4

Canada

Your file Votre riterence

The author has granted a nonexclusive licence allowing the National Library of Canada to reproduce, loan, distribute or sell copies of this thesis in microform, paper or electronic formats.

The author retains ownership of the copyright in this thesis. Neither the thesis nor substantial extracts from it may be printed or otherwise reproduced without the author's permission.
L'auteur a accordé une licence non exclusive permettant à la Bibliothèque nationale du Canada de reproduire, prêter, distribuer ou vendre des copies de cette thèse sous la forme de microfiche/film, de reproduction sur papier ou sur format électronique.

L'auteur conserve la propriété du droit d'auteur qui protège cette thèse. $\mathrm{Ni}$ la thèse ni des extraits substantiels de celle-ci ne doivent être imprimés ou autrement reproduits sans son autorisation.

\section{Canadầ}




\title{
BGP INTER-DOMAIN ROUTING TRAFFIC ANALYSIS
}

\author{
Submitted by Huijun Yang \\ in partial fulfillment of the requirements for the degree of \\ Master of Science \\ Information and Systems Science
}

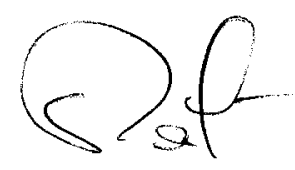

Prof. R. Goubran, Chair, Department of Systems and Computer Engineering

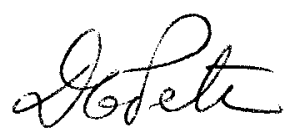

Prof. Dorina Petriu, Thesis Supervisor

Carleton University

September, 2002 


\begin{abstract}
This thesis studies the characteristics of the inter-domain BGP traffic data collected from border routers at major U.S. Internet exchange points in 2001. The following traffic characteristics were analyzed and compared with previously published results (if any where found): trend of BGP traffic volume, ratio of redundant BGP updates, network livability and route stability. New indicators were proposed for characterizing the livability of networks (prefixes), which show whether the Internet instability is due to a large or a small number of networks (prefixes).

Another part of our work was to conduct performance measurements of real BGP routers to study how different factors affect the BGP routing table update, which in turn has an impact on network stability.
\end{abstract}




\section{ACKNOWLEDGMENTS}

Special thanks go to my thesis supervisor, Dr. Dorina Petriu, for her clear guidance, valuable suggestions and kind encouragement. Without her support, I may never have finished my thesis. Also, I would like to thank the members of my committee for their helpful suggestions on the thesis.

Sincere thanks are given to Fahim Sheikh and Safaa Hasan for their valuable advice.

Also, I would like to thank Diwakar Krishnamurthy for his kind help on clustering analysis. 


\section{TABLE OF CONTENTS}

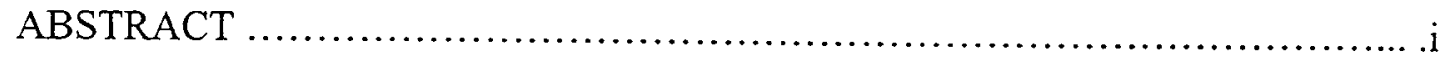

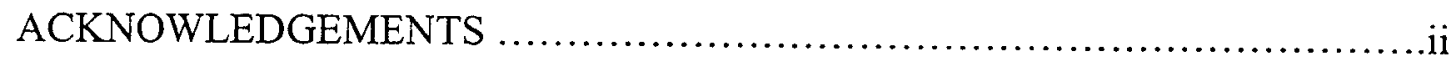

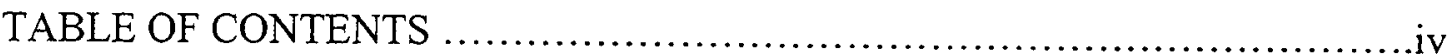

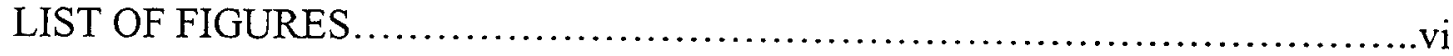

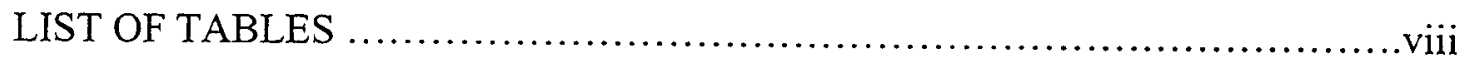

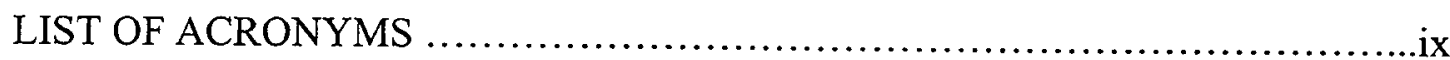

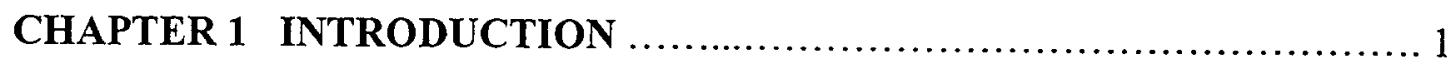

1.1 Motivations and Objectives..........................................

1.2 Thesis Contributions.........................................................

1.4 Thesis Contents. ...........................................................

CHAPTER 2 BACKGROUND AND LITERATURE REVIEW ...............6

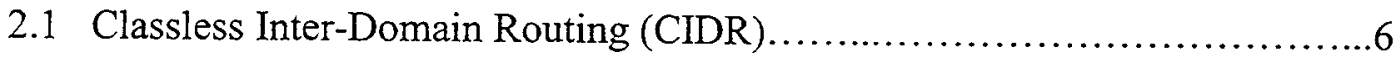

2.2 Border Gateway Protocol (BGP) . .................................... 10

2.2.1 Information Exchange Between Peers............................11

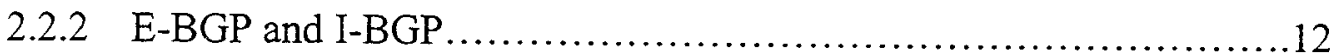

2.2.3 Type of BGP Messages and Attributes..............................13

2.2.4 Routing Process....................................................

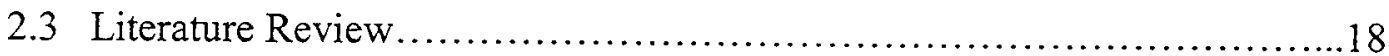

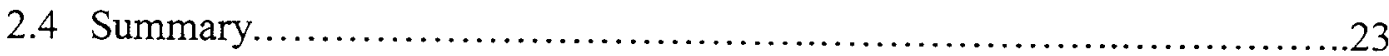

\section{CHAPTER 3 ANALYSIS OF BGP ROUTING TRAFFIC ....................24}

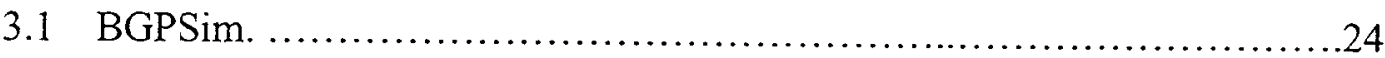

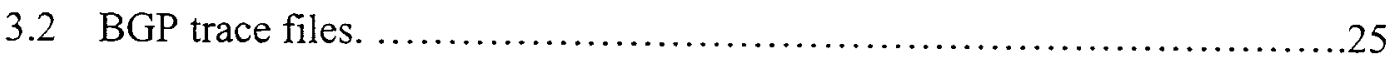

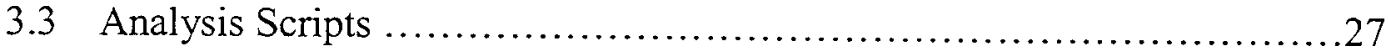

3.3.1 Design of the Perl Statistical Analysis Script. ........................28

3.4 Statistical Analysis of Trace Files..................................... 34 
3.4.1 Analysis of Gross Trends....................................... 35

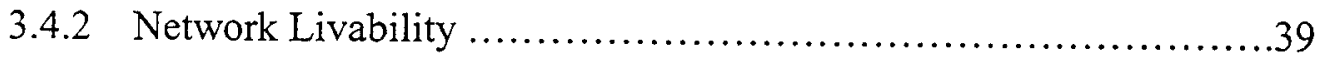

3.4.3 Prefix Distribution. ..........................................42

3.4 .4 Redundancy................................................43

3.4.5 State Change. ...................................................46

3.4.6 Weekly and Daily Pattern.......................................49

$3.5 \mathrm{~K}$-means Clustering Analysis......................................50

3.5.1 Standard Deviation.............................................51

3.5.2 K-means Clustering Algorithm..................................51

3.5.3 Clustering Analysis Results....................................53

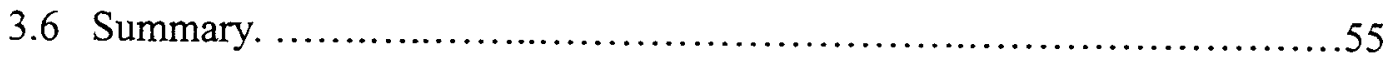

\section{CHAPTER 4 MEASUREMENTS OF BGP UPDATE PROCESSING.............58}

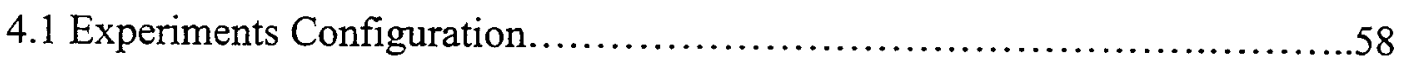

4.2 BGPSim Configuration File Generation Script................................59

4.3 Study of the impact of the policy on TotalProcessingTime. ..................61

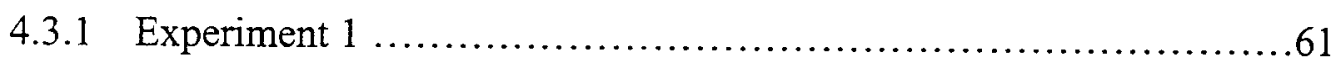

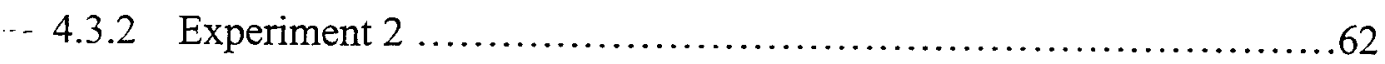

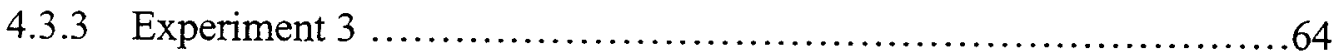

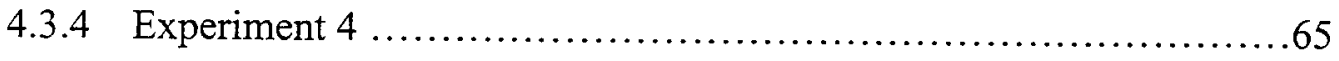

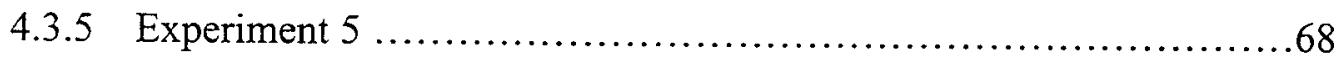

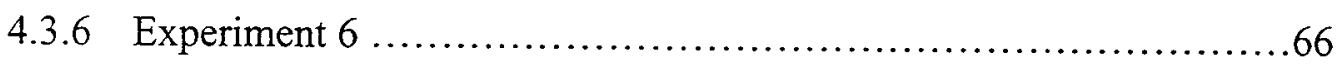

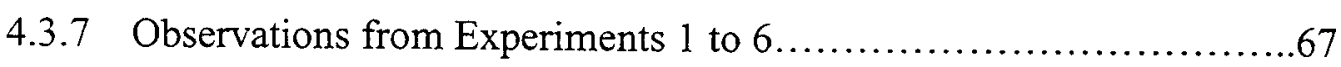

4.4 Study of Effect of Route Stability on TotalProcessingTime..................69

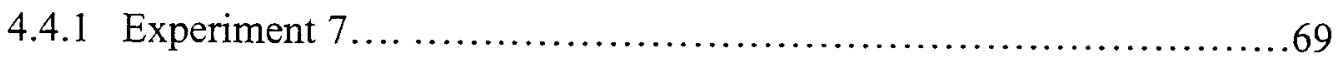

4.4.2 Observations from Experiment 7 ................................. 70

4.5 Study of impact of number of routes advertised on TotalProcessingTime..... 71

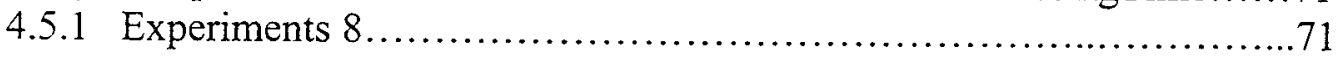

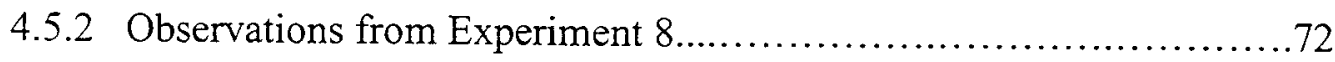

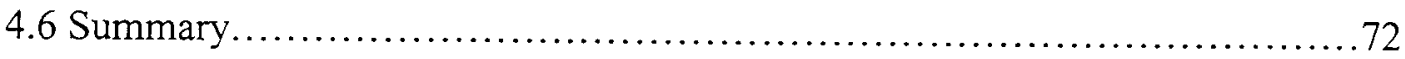




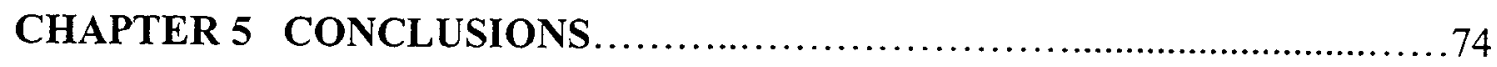

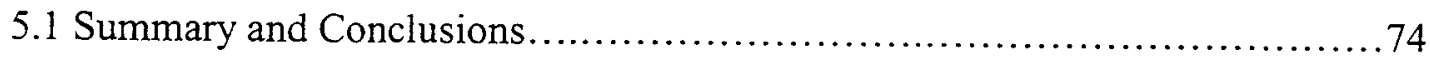

5.2 Future Work........................................................ 77

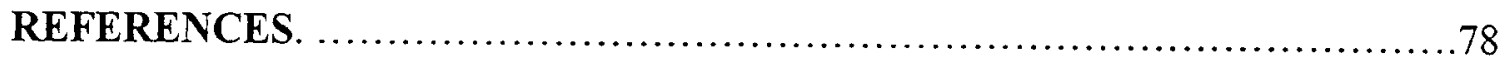




\section{LIST OF FIGURES}

Figure 2.1 Routing Table Without CIDR.....................................

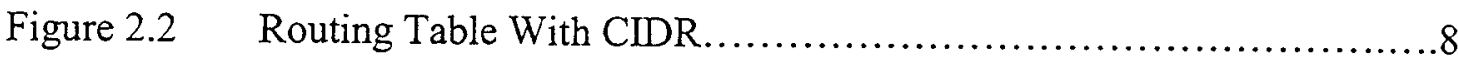

Figure $2.3 \quad$ Border Gateway Protocol Stack................................... 10

Figure $2.4 \quad$ EBGP between AS's. .............................................

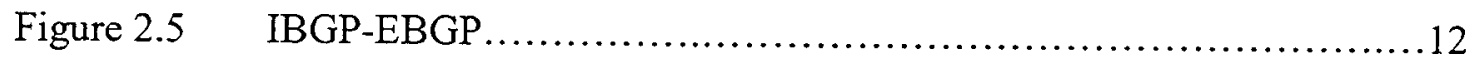

Figure 2.6 BGP Routing Process........................................ 17

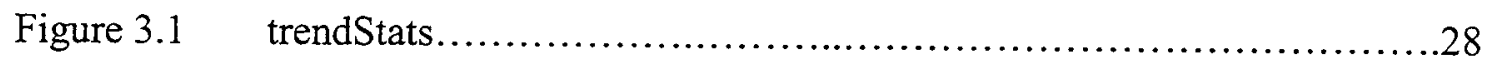

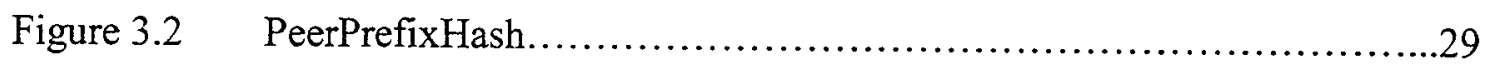

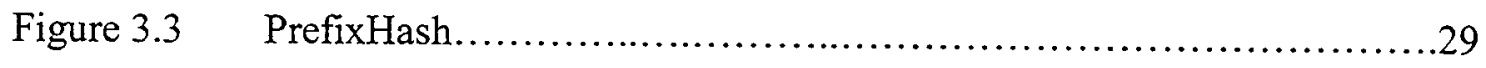

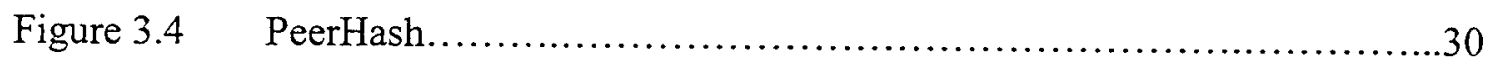

Figure $3.5 \quad$ PrefixLengthHash.............................................. 30

Figure 3.6 Statistical Analysis Perl Script Processing Steps .......................41

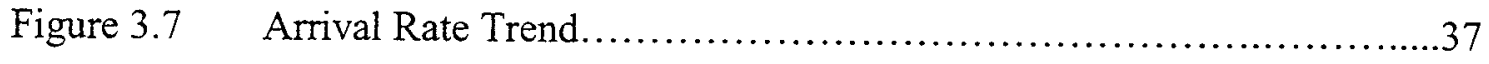

Figure 3.8 BGP Updates Trend......................................... 37

Figure $3.9 \quad$ Unique Prefixes Trend.......................................... 38

Figure $3.10 \quad$ Unique prefixes.................................................

Figure 3.11 Network UpTime...........................................40

Figure 3.12 Network Livability in Jan....................................... 41

Figure 3.13 Network Livability in Feb...................................4

Figure 3.14 Prefix Length distribution........................................ 42

Figure 3.15 Redundant Updates............................................44 
Figure 3.16 Redundant Percentages........................................ 44

Figure 3.17 Redundancy Indicator in Jan.....................................

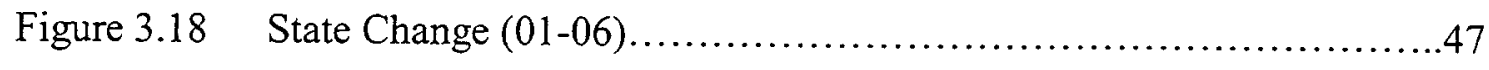

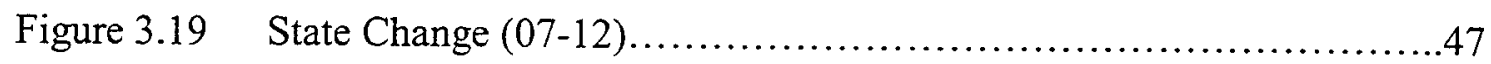

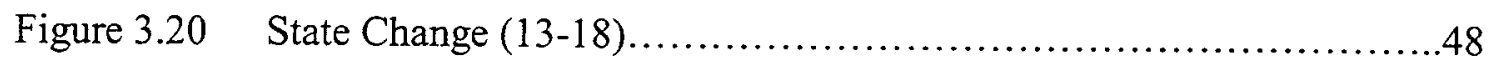

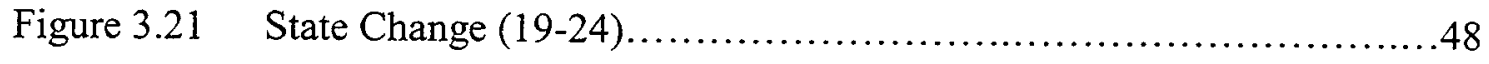

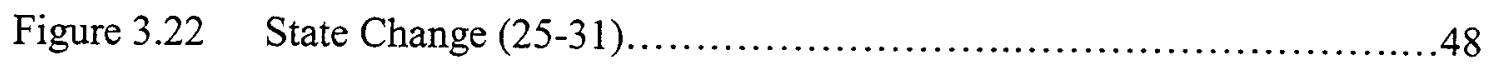

Figure $3.23 \quad$ Weekly Pattern.................................................. 49

Figure 3.24 Days Pattern............................................... 49

Figure $3.25 \quad \mathrm{k}$-means Clustering Algorithm...................................52

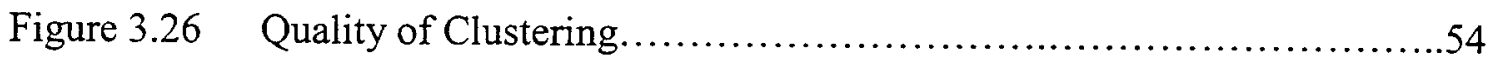

Figure 4.1 Experiment Configuration...................................... 59

Figure 4.2 Impact of Policy on Total Processing Time (1)......................62

Figure 4.3 Impact of Policy on Total Processing Time...........................63

Figure 4.4 Effect of Route Stability on TotalProcessingTime......................70

Figure 4.5 Effect Impact of \#Routes Advertised on TotalProcessingTime..........72 


\section{LIST OF TABLES}

Table $3.1 \quad$ Mean of BGP Updates...................................................

Table 3.2 Route Stability Clustering......................................5

Table 4.1 Impact of Policy on Total Processing Time (1)......................61

Table 4.2 Impact of Policy on Total Processing Time (2).......................62

Table 4.3 Impact of Policy on Total Processing Time (3) ........................64

Table 4.4 Impact of Policy on Total Processing Time (4).......................65

Table 4.5 Impact of Policy on Total Processing Time (5).......................68

Table 4.6 Impact of Policy on Total Processing Time (6).......................69

Table 4.8 Impact of Instability on Total Processing Time.......................70

Table 4.9 Impact of \#Routes Advertised on Total Processing Time.................71 


\section{LIST OF ACRONYMS}

AS

BGP

BGPSim

CIDR

E-BGP

EGP

I-BGP

IGP

IP

IPMA

MRT

NLRI

OSPF

TCP
Autonomous System

Border Gateway Protocol

Border Gateway Protocol Simulator

Classless Inter-Domain Routing

Exterior BGP

Exterior Gateway Protocol

Interior BGP

Interior Gateway Protocol

Internet Protocol

Internet Performance Measurement and Analysis project

Merit Routing Toolkit

Network Layer Reachability Information

Open Shortest Path First

Transport Control Protocol 


\section{CHAPTER 1. INTRODUCTION}

In this chapter, we will discuss the motivation for the thesis, present the contributions of this work, and provide the thesis outline.

\subsection{Motivations and Objectives}

Communication networks play an increasingly important role in industry, business, military and our daily life. As more and more applications rely on communication networks, and especially on the Internet, the demand to provide a robust and reliable network environment is becoming more critical. However, Internet routing instability is a challenging issue facing the Internet engineering community. It is known that a high level of the Internet instability can result in the loss of the connectivity in networks and can lead to the loss of data traffic, which is highly undesirable.

In order to design/produce more reliable and robust Internet devices, it is useful to be able to test such products under realistic workloads. Since it is very difficult to control the load in a real network setting, the measurements are usually done in the lab under workload produced by traffic generators. Understanding how routers and networks respond to variations in load is important for management and capacity planning based on current and projected usage. However, it is very difficult to generate representative BGP routing workload, because the Internet traffic has a number of unusual characteristics that are not very well understood. Our first goal was to capture real inter- 
domain traffic properties in a BGP synthetic workload that can be used for testing BGP routers.

In principle, there are two opposite approaches for generating traffic with real-world characteristics: trace-based approach and analytical approach. The trace-based workload generation uses prerecorded records of past workloads and either samples or replays traces to generate the workloads. On the other hand, analytical workload generation starts with a mathematical model for various workload characteristics and then generates outputs that adhere to the model.

Each approach has its own strengths and weaknesses. The trace-based approach has the advantage that it is easy to implement, and mimics the activity of a known system. However, it treats the workload as a "black box". As a result, it is hard to get insight into the causes for different behaviors. On the other hand, the model-based approach can be challenging to construct for at least three reasons. First, it is necessary to identify the characteristics of the workload that are important to model. Second, the chosen characteristics must be empirically measured. Third, it can be difficult to create a single workload that accurately exhibits a large number of different characteristics.

In our thesis, we take a middle-way approach. We are using an existing tool named BGPSim [Merit99] that is able to simulate complex BGP routing environments with possibly high level of routing changes, provided it is configured with realistic parameters. After being properly configured, BGPSim generates BGP messages that can be fed to a BGP router under study, which believes it is talking to a peer connected to a whole 
network. In order to choose realistic parameters for BGPSim, we use the results of the statistical analysis of real world BGP inter-domain routing traffic performed in the thesis.

In summary, the goals of this research include:

i. Conduct statistical analysis on the raw BGP inter-domain routing data collected from two major public networks exchange points in U.S. Internet backbone and published on the web for the use of researchers in the field [Merit99]. These traces are believed to represent quite well the Internet traffic characteristics [Labovitz97]. We characterize its properties, and analyze some possible causes of anomalous routing behavior. Moreover, the analysis results help us to obtain parameters for the configuration of BGPSim.

ii. Conduct an experimental study on the factors that can negatively impact the BGP routing updates, which in turn have an impact on the BGP routing convergence time. This study is performed by measuring the behaviors of some commercial routers under workload generated by BGPSim.

\subsection{Thesis Contributions}

The contributions of the thesis can be classified in two categories: contributions to knowledge and practical contributions.
A. Contributions to Knowledge 
- Statistical analysis of very large BGP trace files for characterizing the Internet interdomain traffic. The main characteristics that were analyzed and compared with previously published results (if any were found) are the following: a) trend of BGP traffic volume, b) ratio of redundant BGP updates, c) network livability and d) route instability.

- Proposal of new indicators for characterizing the livability of networks (prefixes), which shows the percentage of time a network (prefix) is up over its total lifetime. These indicators give us an idea whether the instability in the Internet is due to a large or to a small number of networks (prefixes).

- Performance measurements of real BGP routers to study how different factors affect the BGP routing table update, which in turn has an impact on network stability. The factors taken into consideration are: a) whether routing policies are applied or not, b) number of updates to be flooded, c) values for the HoldTime and KeepAlive timers, d) number of peers, e) route stability, and f) number of routes to propagate.

B. Practical Contributions

- Implement a Perl analysis program able to handle and analyze very large files of BGP traces. This program can be applied in the future to new sets of data, whenever 
they will become available. This will allow studying the changes in BGP traffic characteristics over time.

- Conduct measurements on an experimental test bed with two BGP routers under a synthetic workload generated by BGPSim. An Expect script was created to automate the reconfiguration of BGPSim necessary for conducting multiple experiments.

\subsection{Thesis Contents}

This thesis comprises six chapters, which are organized as follows:

Chapter 2 provides some background about the BGP protocol and literature review on the Internet traffic analysis.

Chapter 3 describes the experimental measurements we conducted to study the factors that have an impact on the BGP routing table updates. The measurements were done under a synthetic workload generated by BGPSim

Chapter 4 gives an introduction on the analysis program implemented to characterize the properties of the real world BGP routing traffic data, then, introduces the k-means cluster algorithms used in this study, finally, presents the analysis results.

Chapter 5 summarizes our findings and identifies directions for future work. 


\section{CHAPTER 2: BACKGROUND AND LITERATURE REVIEW}

In this chapter, we will describe briefly the CIDR and BGP protocol, then discuss related work on Internet traffic analysis. The focus is mainly on BGP traffic analysis.

\subsection{Classless Inter-Domain Routing (CIDR)}

The Internet is really the interconnection of many individual networks. So the Internet Protocol is basically the set of rules for one network communicating with the others. Each network must know its own Internet address and that of any other networks with which it communicates. To be part of the Internet, an organization needs an Internet network number, which it can request from the Network Information Center (NIC). This unique network number is included in any packet sent out by the network onto the Internet. In addition to the network address or number, information is needed about which specific machine or host in a network is sending or receiving a message. So the IP address needs both the unique network number and a host number, which is unique within the network. The size of the network and host parts depends on the class of the address, and is determined by address' network mask. The network mask is a binary mask with $1 \mathrm{~s}$ in the network part of the address, and 0 in the host part.

To allow for a range from big networks with a lot of computers, to small networks with a few hosts, the IP address space is divided into 5 classes, from A to E. The first byte of the address determines which class an address belongs to:

- Class A addresses begin with $\mathbf{0 x x x}$, or $\mathbf{1}$ to 126 decimal. 
- Class B addresses begin with 10xx, or 128 to 191 decimal.

- Class C addresses begin with $110 x$, or 192 to 223 decimal.

- Class D addresses begin with 1110, or 224 to 239 decimal.

- Class E addresses begin with 1111, or 240 to 254 decimal.

Class $\mathrm{D}$ addresses are reserved for multicasting, and Class $\mathrm{E}$ addresses for future use. They should not be used for host addresses.

As the Internet has evolved and grown in recent years, it has become clear that it will soon face several serious scaling problems. These include:

- Exhaustion of the class-B network address space. One fundamental cause of this problem is the lack of a network class of a size that is appropriate for a mid-sized organization. Class-C, with a maximum of 254 host addresses, is too small, while class-B, which allows up to 65534 addresses, is too large to be densely populated. The result is inefficient utilization of class-B network numbers [RFC1519]. For example: assuming that an organization needs 500 addresses, a single class $\mathrm{C}$ address is not enough (256 hosts). Instead a class B address is allocated ( $\sim 64 \mathrm{~K}$ hosts), which represents a huge waste of the address space.

- Routing information overload. The size and growth rate of the routing tables in Internet routers is beyond the ability of current software to effectively manage [RFC1519].

- Eventual exhaustion of IP network numbers. It has become clear that the first two of these problems are likely to become critical in the near term. Classless InterDomain Routing (CIDR) attempts to deal with these problems by defining a 
mechanism to slow the growth of routing tables and reduce the need to allocate new IP network numbers. It does not attempt to solve the third problem, which is of a more long-term nature, but instead attempts to ease enough of the short to mid-term difficulties to allow the Internet to continue to function efficiently while progress is made on a longer-term solution [RFC1519].
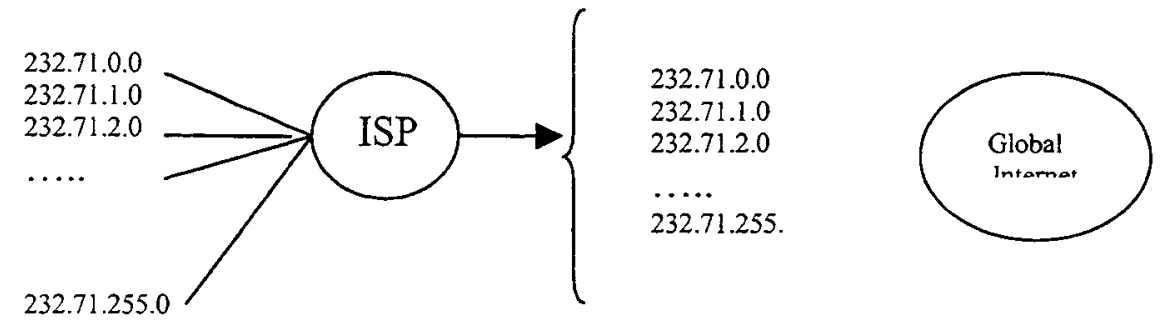

Figure 2.1 Routing Table Without CIDR
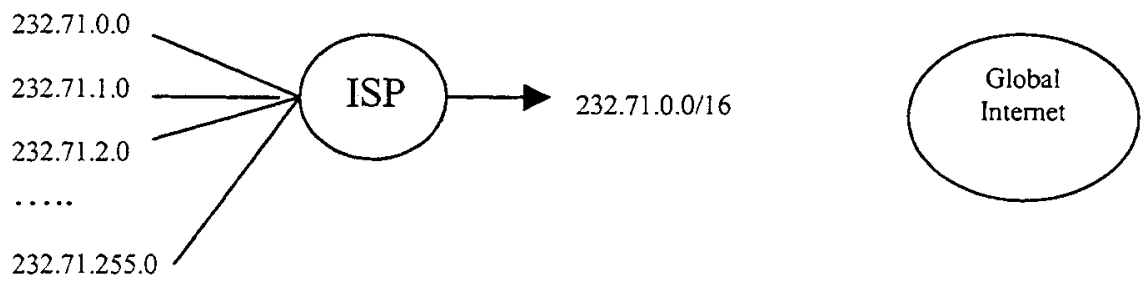

Figure 2.2 Routing Table Without CIDR

CIDR gets rid of the class concept, and allows networks to be assigned on arbitrary bit boundaries. It permits arbitrary sized masks, i.e. $178.24 .14 .0 / 23$ is valid. It requires explicit masks to be passed in routing protocols. For example, an organization can be allocated a single $/ 23$ address (equivalent of 2 class C's). Thus, it solves the first problem for IP addresses assignment. 
Comparing Figure2.1 with Figure 2.2, it is very easy to tell the difference. Without CIDR an IP address corresponds to one of the 5 classes, from A to E, described above. With CIDR, the IP address usually is expressed in the following format:

< address/prefix length >

where the prefix length denotes the upper bits of the IP address.

The <address/prefix length> pair defines an address block. For example:

$$
\begin{aligned}
& -232.71 .0 .0 / 16 \Rightarrow[232.71 .0 .0-232.71 .255 .255] \\
& -198.31 .0 .0 / 13 \Rightarrow[198.31 .0 .0-198.31 .255 .255]
\end{aligned}
$$

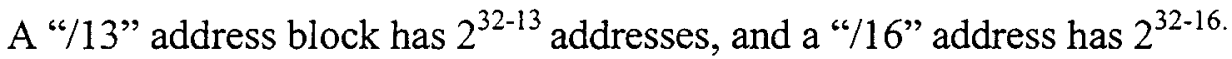

CIDR is using aggregation to provide routing for a large number of customers by advertising one common prefix, as shown in Figure 2.2. This is possible because of the hierarchical nature of addressing. Such aggregation can summarize routing information, and reduce the size of routing tables, allowing at the same time to maintain connectivity.

Because prefixes of arbitrary length are allowed, overlapping prefixes can exist.

For example, if a router hears 124.39.0.0/16 from one neighbor and 124.39.16.0/24 from another neighbor, it forwards the packets according to the most specific forwarding information, called the "longest prefix match". Packet with the destination 124.39.16.32 will be forwarded using the "/24" entry, whereas those with the destination 124.39 .82 .45 will be forwarded using " $/ 16$ " entry. 


\subsection{Border Gateway Protocol (BGP)}

An Autonomous System (AS) is a set of routers under a single technical administration. The whole Internet is composed of many ASs. The routing inside and between ASs is done by different protocols. The Border Gateway Protocol (BGP) is a layer 3 routing protocol that can be used in the Internet as both inter-ASs and intraASs routing protocol. The primary function of a BGP speaking system is to exchange network reachability information with other BGP systems [RFC1771].

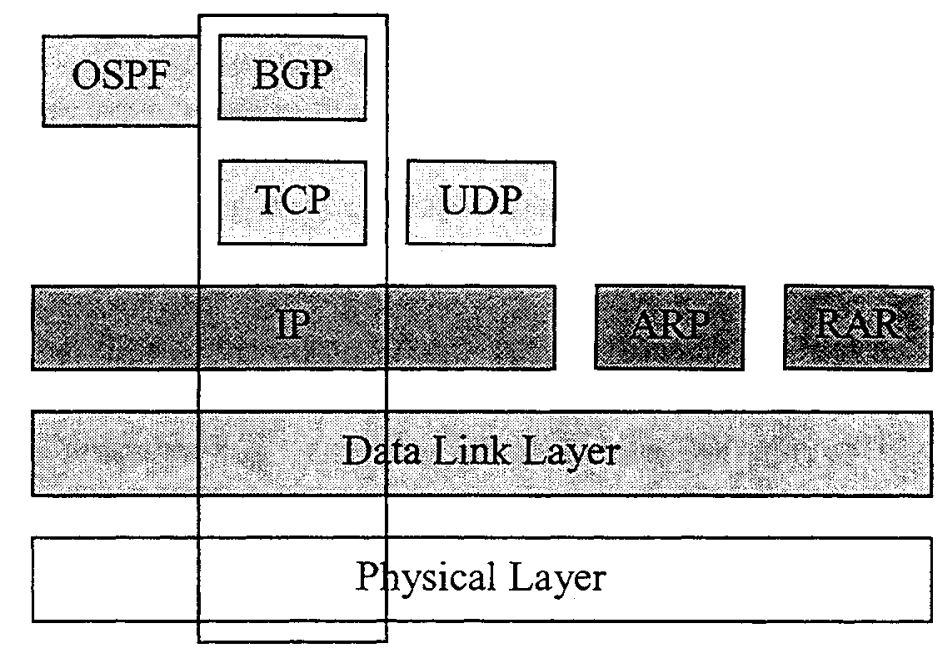

Figure 2.3 Border Gateway Protocol Stack

This network reachability information includes information on the list of ASs that the messages carrying this information traverse. An AS is using an interior gateway 
protocol and common metrics to route packets within the AS, and using an exterior gateway protocol to route packets to other ASs. [RFC1771] An AS is identified by an AS number. This information is sufficient to construct a graph of AS connectivity from which routing loops may be pruned and some policy decisions at the AS level may be enforced.

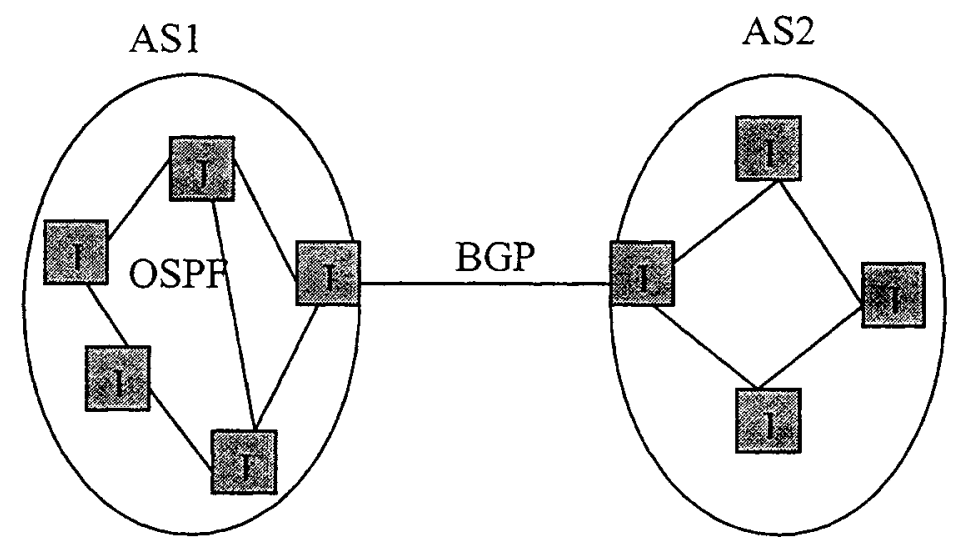

Figure 2.4 EBGP between AS's

\subsubsection{Information Exchange Between Peers}

Any two routers that have formed a TCP connection in order to exchange BGP routing information are called peers, and they are also known as neighbors. BGP peers will initially exchange their full BGP routing tables. From then on, only incremental updates are sent as the routing table changes. BGP keeps a version number of the BGP table and it should be the same for all of its BGP peers. The version number will change whenever BGP updates the table due to some routing information changes. KEEPALIVE packets are sent to ensure that the connection is 
alive between the BGP peers and NOTIFICATION packets are sent in response to errors or special conditions. BGP routes have two primary origins: transit routes and internally originated routes. A transit route is a prefix learned via an external BGP peering session and re-advertised to one or more external BGP peers. An internal route is a prefix learned via an internal, inter-domain routing protocol running within the autonomous system. Internal routes may be statically configured on internal or border routes [RFC1771].

\subsubsection{E-BGP and I-BGP}

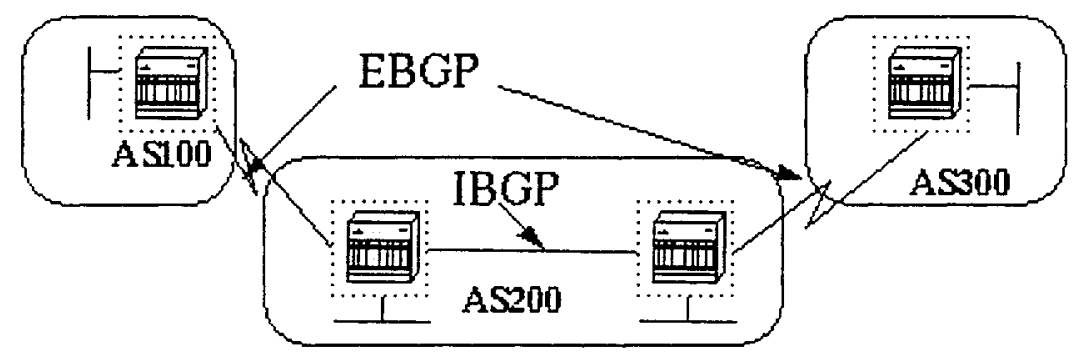

Figure 2.5 IBGP-EBGP

It is necessary to ensure reachability for networks within an AS before sending the information to other external ASs. This is done by a combination of Internal BGP peering between routers inside an AS and by redistributing BGP information to Internal Gateway Protocols running in the AS. 
When BGP is running between routers belonging to two different ASs, it is called EBGP (Exterior BGP) and when is running between routers in the same AS it is called IBGP (Interior BGP).

E-BGP neighbors have a restriction on being physically connected. BGP drops any updates from its external BGP peer if the peer is non-connected. On the other hand, neighbors within the same autonomous system have no restrictions whether the peer routers are physically connected, as long as there is IP connectivity between the two neighbors.

\subsubsection{Type of BGP Messages and Attributes}

According to [Stewart99], there are different types of BGP messages as following. OPEN: is the first message sent after the TCP connection is established. The purpose is for each endpoint to identify itself to the other and to agree on protocol parameters, such as timers.

UPDATE: is the primary message used to communicate routing information between two BGP speakers (routing processes). There are two types of Update messages: one is Withdrawal, which withdraws a previously advertised prefix. The other one is Announcement, used to advertise a new prefix to the BGP neighbors. 
NOTIFICATION: is used to signal the presence of an error during the life of a BGP session before the underlying TCP connection is closed.

KEEPALIVE: BGP neighbors send KEEPALIVE to each other to confirm that the connection is still active.

BGP-4 [RFC1771] provides a new set of mechanisms for supporting classless interdomain routing. These mechanisms include support for advertising an IP prefix and eliminate the concept of network "class" within BGP by using CIDR. BGP-4 also introduces mechanisms, which allow aggregation of routes, including the aggregation of AS-PATH. To characterize the set of policy decisions that can be enforced using BGP, one must focus on the rule that a BGP speaker advertise to its peers (other BGP speakers which it communicates with) in neighboring ASs only those routes that it itself uses. This rule reflects the "hop-by-hop" routing paradigm generally used throughout the current Internet. [Stewart99]

It is assumed that BGP runs over a reliable transport protocol. This eliminates the need to implement explicit update fragmentation, retransmission, acknowledgement, and sequencing. Any authentication scheme used by the transport protocol may be used in addition to BGP's own authentication mechanisms. The error NOTIFICATION mechanism used in BGP assumes that the transport protocol supports a "graceful" close, i.e., that all outstanding data will be delivered before the 
connection is closed. The key features of the protocol are the notion of path attributes and aggregation of network layer reachability information (NLRI).

Path attributes provide BGP with flexibility and expandability. Path attributes are partitioned into well known and optional. The provision for optional attributes allows experimentation that may involve a group of BGP routers without affecting the rest of the Internet. New optional attributes can be added to the protocol in the same fashion as new options are added to the Telnet protocol, for instance. One of the most important path attributes is the AS-PATH. AS reachability information traverses the Internet, this information is augmented by the list of autonomous systems that have been traversed thus far, forming the AS-PATH. The AS-PATH allows straightforward suppression of the looping of routing information. In addition, the AS-PATH serves as a powerful and versatile mechanism for policybased routing. [RFC1771]

BGP-4 enhances the AS-PATH attribute to include sets of autonomous systems lists as well. This extended format allows the aggregate routes to carry path information from the more specific routes used to generate the aggregate.

As we know, the routing protocols usually can be classified into two categories: distance vector and link state. However, BGP uses an algorithm that cannot be classified as either a pure distance vector, or a pure link state. Carrying a complete AS path in the AS-PATH attribute allows reconstructing large portions of the overall 
topology. That makes it similar to the link state algorithms. Exchanging only the currently used routes between the peers makes it similar to the distance vector algorithms.

To conserve bandwidth and processing power, BGP uses incremental updates, where after the initial exchange of complete routing information, a pair of BGP routers exchanges only changes (deltas) to that information. Technique of incremental updates requires reliable transport between a pair of BGP routers. To achieve this functionality BGP uses TCP as its transport protocol.

In addition to incremental updates, BGP-4 has added the concept of route aggregation so that information about groups of networks may be represented as a single entity. This feature can abstract some routing instability at a certain level.

\subsubsection{Routing Process}

BGP is a fairly simple protocol, which explains why it is so flexible. Routes are exchanged between BGP peers via UPDATE messages. BGP routers receive the UPDATE messages, run some policies or filters over the updates, and then pass on the information to other BGP peers. In addition to passing along routes, a BGP router may originate routing updates to advertise networks that belong to its own autonomous systems. Valid local routes originated in the system, and the best routes 
learned from BGP peers are then installed in the IP routing table. The IP routing table is used for the final routing decision.

Each BGP speaker, also called BGP routing process, has different pools of routes and different policy engines applied to the routes. The model of the BGP process as shown as Figure 2.4, involves the following components:

- A pool of routes that the router receives from its peers

- An Input Policy Engine that can filter the routes or manipulate their attributes

- A decision process that decides which routes the router itself will use

- A pool of routes that the router itself uses

- An Output Policy Engine that can filter the routes or manipulate the attributes

- A pool of routes that the router advertise to other peers

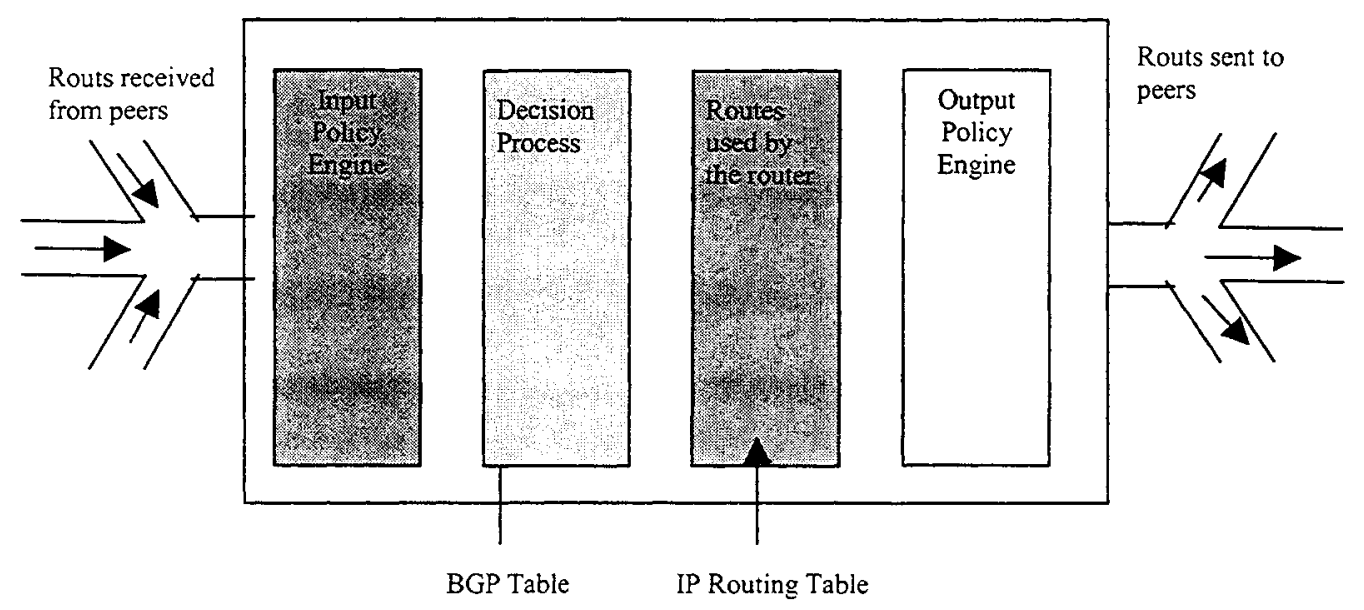

Figure 2.6 BGP routing process 


\subsection{Literature Review}

In the recent years, Internet has experienced a rapid growth. As more and more applications rely on it, the demand to provide a robust, scalable, and reliable Internet infrastructure has become more and more critical. However, one of the problems faced by the Internet engineering community is the Internet routing instability, which is defined as the rapid change of network reachability information. [Labovitz97]. It is known that a high level of Internet instability can lead to the loss of connectivity in Internet, slow the routing convergence, produce packets loss, and also waste the network resources, such as $\mathrm{CPU}$, memory, etc. In the past few years, there has been a lot of effort to identify and eliminate the causes for Internet routing instability. In this chapter, we will give a brief overview of the work related to the inter-AS routing instability.

A study on the dynamic of Internet routing information, conducted by Bilal Chinoy, [Chinoy93] was one of the very first studies in this area. He investigated the dynamics of routing information flow in the NSFNET backbone network. Data was collected on a 12hour period on August 1992 from all the NSFNET nodes on the T1 backbone. The following aspects were investigated:

1. Efficiency of routing protocols: how much of the routing updates are useful?

2. The size of networks that becomes unreachable.

3. Frequency distribution of state transitions of various networks (a state transition is a change from unreachable state to reachable or vice versa.)

It was found that, at that time, both EGP and BGP were used as inter-AS routing protocols. According to their results $90 \%$ of the updates of EGP contained no new 
information. Most routing changes occurred at the edges of the network. Network outages span a large range of time, from minutes to hours. Only a few of the networks made frequent connectivity transitions.

Robert Malan, Craig Labovitz, and Farnam Jahanian conducted several related studies [Labovitz97] [Labovitz99] on Internet routing instability by analyzing the dynamic Internet routing information. They studied the network inter-domain routing information exchanged between backbone service providers at the major U.S public Internet exchange points. Their goal was similar to the research on end-to-end routing behavior, [Paxson96] which aims to inspect the routing instability. As already mentioned, routing instability causes packet loss, increased network latency, and increased convergence time. To analyze the routing behavior, the authors of [Labovitz97] used BGP routing messages generated by border routers at five of the major public exchange points of U.S. during a nine months period. Since the traffic volume through the public exchange points is very large, the analysis provided significant conclusions about the routing behavior of the Internet. This data reflects the stability of inter-domain routing, as well as the changes in topology or policy between autonomous systems. Intra-domain instability information is observed by analyzing the BGP information.

In [Labovitz97] the authors found that the number of BGP updates exchanged in the Internet core is much larger than it was expected at the time. One of the reasons was that the majority of the BGP routing updates were pathological or redundant, which are repeated transmissions of identical routing information that do not reflect real topology or 
policy changes. Even though redundant updates may not affect the network topology directly, big volumes of them add a very large overhead on the routing component. In conclusion, the network resources are wasted, and the routing component may even be reduced to very low levels if the volume of updates becomes overwhelming. This results directly in instability of the Internet [Labovitz97]. The authors also claimed that Internet instability is not dominated by a small set of ASs or prefixes, but is well distributed. According to [Labovitz97], some instability is caused by some features of the router architecture. For example, the route-caching architecture that was commonly used in many Internet routers or switches could potentially cause some Internet instability. In this architecture, routers or switches maintain a routing table cache. When packets for a particular destination come in to a router or switch, the router will look up first the cache. If an entry for this destination is found in cache, the packet will be forwarded right away based on the information stored in the cache entry. If no entry for this destination is found in cache, then the router will have to look up the forwarding table to decide how to forward the packet. If the forwarding table lookup is successful, the packet will be forwarded accordingly, and the information for the respective destination will be stored in cache. This way, the next packets for the same destination will be forwarded fast. Normally, with this mechanism, the forwarding process can become much faster. However, if the network instability increases, or the cache size is not big enough, the number of cache misses, respectively of cache updates will increase. This may eat up a lot of CPU processing time, and if the traffic volume happens to be big, it will make the CPU so busy that eventually network connections may be dropped. This will increase the degree of network instability even more. 
The same authors extended in [Labovitz99] the work reported in [Labovitz97]. They collected more data at the same exchange points and were able to verify that as a result of changes to specific router vendor software, suggested by the analysis from [Labovitz97], the volume of routing updates had decreased by an order of magnitude. The decrease has happened because of the reduction of pathological withdrawals. The authors also proposed new potential changes to router software that could decrease the volume of pathological withdrawals by an additional 30 percent or even more.

Overall, the work reported in [Labovitz99] gives a very good idea of the complexity of Internet traffic with facts and numbers with a lot of meaning. However, one confusing point in the paper is the seemingly contradicting statements that a) no particular routes are causing the instability and b) only $3-20 \%$ of the routes generated the wrong updates.

Delayed routing convergence has been recognized as a well-known factor that can result in Internet instability, which can lead in turn to end-to-end Internet paths experiencing intermittent loss of connectivity, as well as increased packet loss and latency. There have been several studies looking at the routing instability from the routing convergence perspective.

Aman Shaikh's and Lampros Kalampoukas' study on the routing stability in congested networks, [Shaikh00] found that the loss of the routing protocol messages due to network congestion can cause peering session failures in routers, leading to route flaps and routing instabilities. They studied the effects of traffic overload on routing protocols by 
quantifying the stability and robustness properties of two common Internet routing protocols, OSPF and BGP, when the routing control traffic is not isolated from data traffic. Their analysis results show that increased queuing and propagation delays adversely affect BGP's resilience to convergence, which in turn can have impact on the Internet instability.

A study conducted by T.G. Griffin and Gordon Wilfong on the BGP convergence properties [Griffin99] shows that BGP is a path-vector protocol that allows each AS to override distance-based metrics with policy-based metrics for choosing the best routes. It is possible for a group of ASs to independently define BGP policies that together lead to BGP protocol oscillations that never converge on a stable routing. One approach to addressing this problem is based on static analysis of routing policies to determine if they are safe. The authors explore the worst-case complexity for convergence-oriented static analysis of BGP routing policies. They present an abstract model of BGP and use it to define several global sanity conditions on routing policies that are related to BGP convergence/divergence. For each condition they show that the complexity of statically checking it is either NP-complete or NP-hard [Griffin99].

Craig Labovitz and Ahba Ahuija also studied the impact of Internet policy and topology on delayed routing convergence in [Labovitz01]. They found that Internet backbone routers may take minutes to reach a consistent view of the network topology after a fault. The time for end-to-end Internet convergence depends on the length of the longest possible backup autonomous system path between a source and destination node. Finally, 
they propose some possible modifications to BGP and provider routing policies, which would improve inter domain routing convergence. [Labovitz01]

\subsection{Summary}

In this chapter, we presented a brief background for the BGP protocol. Then we discussed the Internet instability issue, and reviewed related work on Internet routing traffic analysis. 


\section{CHAPTER 3. ANALYSIS OF BGP ROUTING TRAFFIC}

\subsection{BGPSim}

The original motivation for the thesis research was to measure the performance of a certain BGP router under "reasonable" load. Since such a load cannot be easily produced in a small experimental test bed, the traffic generator tool BGPSim was used [Merit99]. BGPSim is a BGP4+ traffic generator/simulator, one of the components from the MRT toolkit developed by Merit Networks Inc. BGPSim can be used to explore the scaling properties of routing protocols and routers, as long as is properly configured. A text configuration file that define groups of networks with a variety of network characteristics, such as number of routes, topological connectivity (AS-PATH, LOCAL_PREF, COMMUNITY), routing policies, etc. is required as input to BGPSim. For more detailed information on the usage of the BGPSim, and on how to construct a configuration file, please refer to [Merit99].

BGPSim can provide a means of generating traffic conditions that equal or even exceed the worst-case scenarios seen to date in the Internet core. Such traffic can be used as a stress test for new BGP routers, and would be very difficult to achieve without BGPSim, just by using a local test bed. However, from the experience of using BGPSim during the thesis research, we found that it presents its own challenges. We found that it is not easy to choose the "right" parameters for the BGPSim configuration file, such that the synthetic workload generated exhibits accurately characteristics of the real-world routing traffic. We needed to understand better the characteristics of the real world BGP traffic in 
order to set up appropriate BGPSim configuration files. This was the original motivation for analyzing real world traces of BGP traffic, as presented in the following sections.

\subsection{BGP Trace Files}

Our analysis in this work is based on raw BGP traffic data collected from the experimental instrumentation of the core exchange point of the Internet infrastructure. The measurements were collected by IPMA, Merit Networks and made available on the web at ftp://ftp.merit.edu/statistics/ipma/data/. The Routing Arbiter project's route servers, deployed on IPMA probe machines at U.S. inter-exchange points, Mae-East, Mae-West, AADS, PacBell, collects routing information and dumps the data to files in a predetermined, time-stamped format. Raw traffic data is gathered using passive listening tools, which do not negatively impact the network. In this work, our analysis simply focuses on the logs of Mae-East and Mae-West exchange points. The reason we choose these two exchange point as the study entities is because they are two of the largest public exchange routers. Each hosts currently over 60 service providers, including MCI, SPRINT, BBN, ANS etc, and pretty much represents the most demanding conditions under which a BGP router can work. The producers of BGP routers are certainly interested in testing their products under traffic conditions similar to those experienced by these two exchange points.

Each trace files is 15 -minute log of BGP activity and has an average size of $15 \mathrm{k}$. The data is encoded in a special binary file format and includes the raw BGP packet information received by the router. A fixed, 12-byte non-standard header is prepended that records 
time, source, and destination attached to each BGP packet. The remainder of the packet is an exact copy of the BGP packet received over the wire. We use the utility ROUTE_BTOA, which is part of MRT toolkit, to convert the raw data from the binary format into machine-readable ASCII format, which can be easily parsed by Perl scripts for automated analysis. The format is as follows [Merit99]:

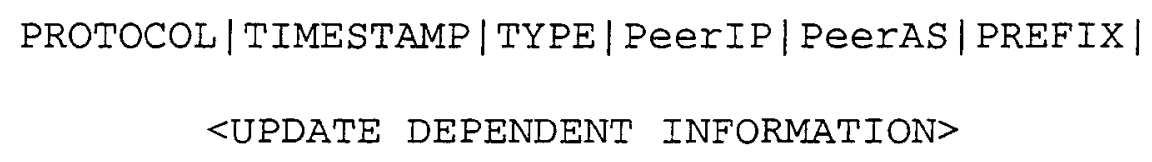

For BGP announcements, <UPDATE DEPENDENT INFORMATION>contains:

ASPATH | ORIGIN | NEXTHOP | LOCAL_PREF | MED | COMMUNITY

where, according to $[\mathrm{RFCl} 171]$ the components have the following meaning:

- ASPATH: the AS system path of the update has traversed.

- ORIGIN: IGP, EGP, or unknown.

- NEXTHOP: is the IP address of the border router that should be used as the next hop to the destinations listed in the UPDATE message.

- LOCAL_PREF: is a BGP-4 attribute that a given BGP speaker sends to the other BGP speakers located in its own autonomous system.

- MED: acronym for MULTI_EXIT_DISC, can be used on inter-AS links to discriminate among multiple exit or entry points to the same neighboring AS.

- COMMUNITY: is a group of destinations (i.e. prefixes) that share some common attribute. [RFC1998] 
Below is an example of the machine-readable format of BGP packets:

BGP | $1009861200|A| 198.108 .60 .7 \mid 1239 \quad 1800 \quad[1755$

43] | IGP| $192.246 .146 .0 / 24|0| 0|| N A G||$

$\mathrm{BGP}|1009873124| \mathrm{W}|198.108 .60 .7| 1239 \quad 1800\left[\begin{array}{lll}1755 & 43\end{array}\right] \mid 192.246 .146 .0 / 24$

The trace files are not available for each and every day. Sometimes the data was not recorded due to the failure of the equipment, or other causes. For our analysis, we used trace files from Mae-East for the year 2001, which were available only for the following nine months: January, February, May, July, August, September, October, November, and December. Unfortunately, we could not find any 2002 data for Mae-East. For a part of the study we used more recent data (January 2002) from another large exchange point, namely Mae-West.

\subsection{Analysis Scripts}

As we mentioned before, there are 96 BGP raw data log files per day, and the average size of each file is about $15 \mathrm{~K}$. So the size of the data for the nine months period we analyzed for Mae-East is a huge amount $(\sim 390 \mathrm{MB})$. It requires not only a lot of physical disk to store, but also scalable software to process it. Applying statistical analysis on that huge amount of data is very challenging. It is interesting to mention that in the very early phase of our research, we failed to assess correctly the size of the data we were trying to study. At first, we designed and implemented the software to process and analyze the data in $\mathrm{C}++$ with Objected-Oriented concepts. We created an object for each and every BGP message, and tried to store all the information found in the trace for further analysis. We used part of the raw data to test it, and it seemed to work well. But 
when we applied it on more data, we realized that we could not process data for more than 3 days at once. In order to solve this problem and make the analysis program able to handle larger amounts of raw data, we had to redesign the software. Instead of storing first all the information in objects, and doing the statistical analysis afterwards, we decided to apply the statistical analysis "on the fly" while processing the data. Also, we found out that Perl is has more powerful features than $\mathrm{C}++$ for extracting data from text files, thus we chose Perl as the language for re-implementing the analysis software. In addition, because the original raw data is stored in binary format, we had to convert all the data from binary into ASCII format before processing it.

Fortunately, with the new Perl program (approximately 1000 lines of code), we were able to process data for up to a month period at once. As shown in the following sections, most analysis results are given on a monthly basis. In the following session, we will describe briefly the design of the Perl analysis program.

\subsubsection{Design of the Perl Statistical Analysis Script}

The main data structures of the Perl script are shown in Figures 3.1 to 3.5.

\section{TrendStats:}

$$
\begin{aligned}
& \text {-\#ofA } \\
& \text {-\#ofW } \\
& \text {-TotalUpdates } \\
& \text {-ArrivalRate }
\end{aligned}
$$

Figure 3.1 TrendStats data structure 


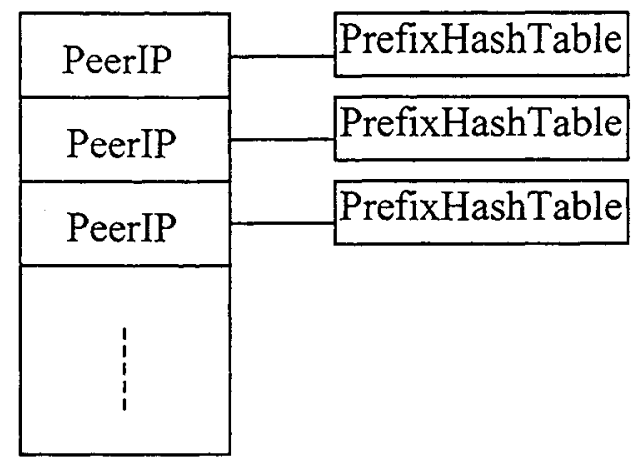

Figure 3.2 PeerPrefixHash Table

PeerPrefixStats:

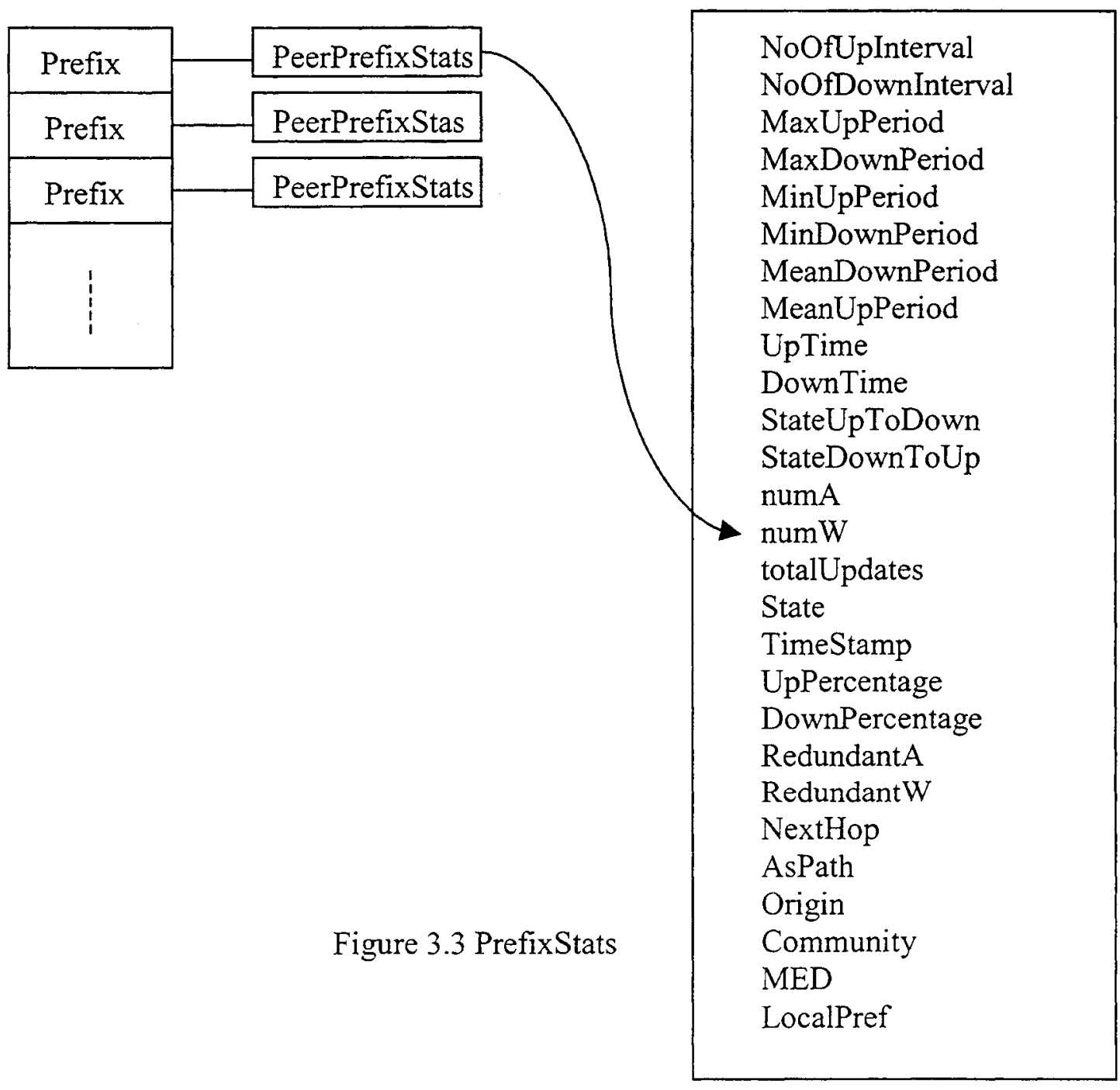




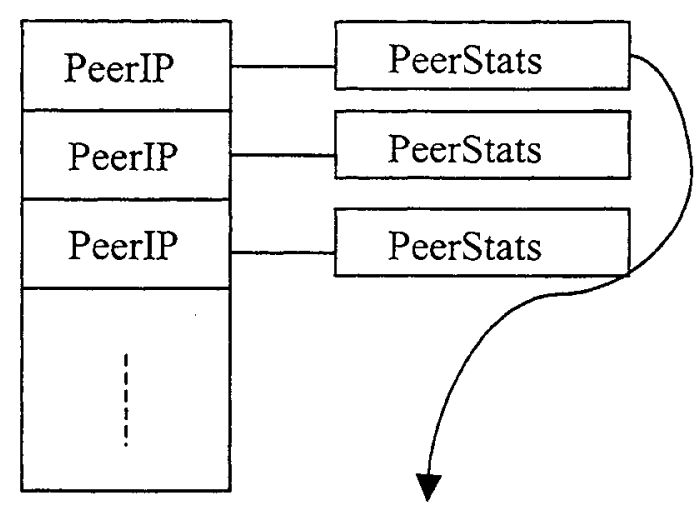

PeerStats:

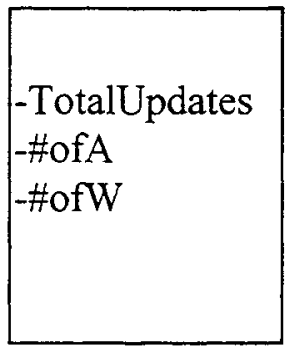

Figure 3.4 PeerHash Table

PeerLengthStats:

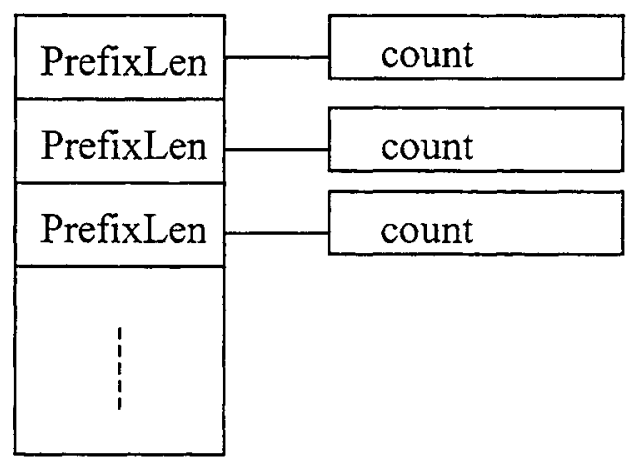

Figure 3.5 PrefixLengthHash Table

As shown in the figures above, the statistical results has been classified into different categories:

- TrendStats show the overall trends statistics: 
- \#ofA: total number of BGP Announcements in trace files being processed

- \#ofW: total number of BGP Withdrawals in trace files being processed

- TotalUpdates: total number of BGP updates in trace files being processed

- PeerPrefixStats show the statistical results corresponding to a route:

- NoOfUpInterval: how many UP intervals are found in the study period

- NoOfDownInterval: how many DOWN intervals are found in the study period

- MaxUpPeriod: the longest time period when the route is feasible

- MaxDownPeriod: the longest time period when the route is infeasible

- MinUpPeriod: the shortest time period when the route is feasible

- MinDownPeriod: the shortest time period when the route is infeasible

- MeanDownPeriod: the mean value of down intervals for this route

- MeanUpPeriod: the mean value of up intervals for this route

- Uptime: the accumulated time when the route is up

- Downtime: the accumulated time when the route is down

- UpPercentage: the percentage of time in the study period when the route is up

- DownPercentage: the percentage of time in the study period when the route is down

- RedundantA: redundant BGP Announcement

- RedundantW: redundant BGP Withdrawal

- StateFromUpToDown: number of times when the route goes down 
- StateFromDownToUp: number of times when the route goes up

- \#ofA: number Announcements for the route

- \#ofW: number of Withdrawals for the route

- TotalUpdates: total number of BGP updates for the route

- State: whether the route is up or down.

The rest of the fields are to store BGP update information, which is needed for redundant updates related statistics. As the following fields have been explained in the section 3.2, we would not describe them here again.

- Timestamp

- Nexthop

- AsPath

- Community

- Origin

- MED

- LocalPref

- PeerStats show the statistical result corresponding to a peer:

- \#ofA: total number of BGP Announcements received from a peer

- \#ofW: total number of BGP Withdrawals received from a peer

- TotalUpdates: total number of BGP updates received from a peer

- PrefixLengthCount show the number of prefixes corresponding to particular prefix length

- count: how many updates on the prefix with a particular prefix length. 
We will discuss some of the above statistics in more details when we present the results in later sections. The Perl script is designed to continuously process all the trace files located in the "current" directory, in the format described in section 3.2. The processing steps are being summarized in Figure 3.6.

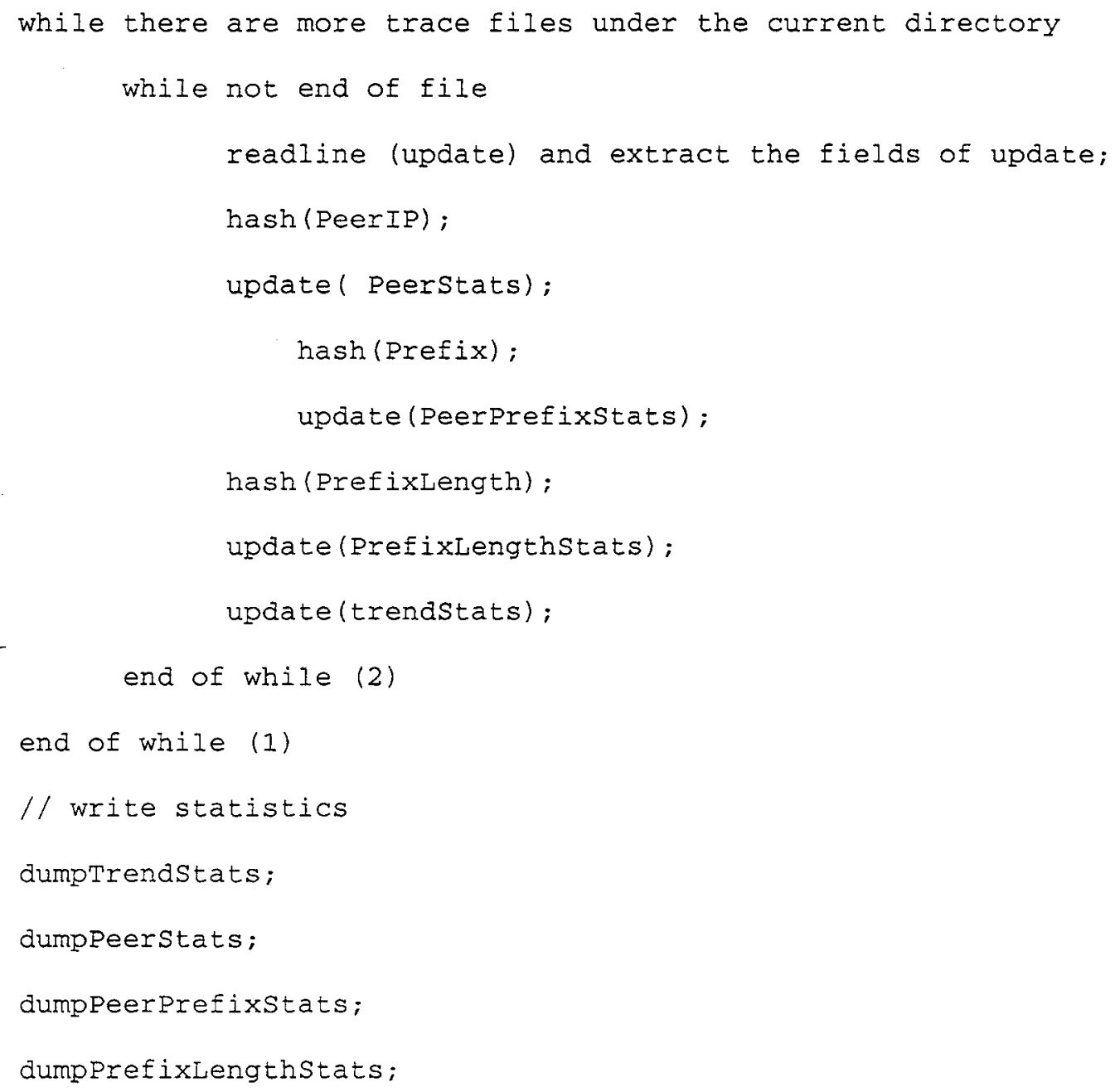

Figure 3.6 Statistical Analysis Perl Script Processing Steps 


\subsection{Statistical Analysis of the Trace Files}

Before starting the analysis for 2001, we expected to see an increase in the volume of the BGP traffic from the 1996 levels reported in [Labovitz97]. Such an increase was expected because the Internet was larger and more complex in 2001 than in 1996. However, the results are contrary to our original expectation. Comparing our results with those from [Labovitz97], we realized that by 2001, the volume of inter-domain routing messages in the Internet core has decreased dramatically by an order of magnitude with respect to the 1996 level. Throughout 1996, the Mae-East exchange point averaged 3 to 5 million BGP updates every day. By the summer of 1998, the aggregate number of BGP updates has dropped to several hundred thousands per day [Labovitz99]. As we found in our study, by December 2001, it had dropped to about 0.3 million per day. As stated in [Labovitz99], the reduction in BGP traffic is mainly due to the following factors:

- Many vendors have deployed dampening algorithms. These algorithms "holddown", or refuse to believe, updates about routes that exceed certain parameters of instability. A router will not process additional updates for a dampened route until a preset, administratively configurable period of time has elapsed.

- High level of prefix aggregation.

A high level of aggregation will result in a small number of globally visible prefixes, and theoretically a greater stability in the prefixes that are announced.

On top of the above reasons given in [Labovitz99], we believe that the following factors also contribute to the stability of the inter-domain routing:

- A significant drop in the number of pathological BGP withdrawals, which we found in our analysis and will discuss later in more detail. 
- Software/hardware bugs fixed by various vendors.

- With the new technologies continuously applied to routers/switches, the capacity and robustness of the equipment has increased dramatically in the past few years.

However, even though we have seen significant improvements in terms of Internet stability in 2001 compared 1998 due to the reasons listed above, our research has shown that nowadays the Internet still continues to exhibit a relatively high level of routing instability. A high percentage of pathological routing behavior is still found in 2001; for example, we found that $56 \%$ of the BGP updates were redundant (i.e., did not reflect real topology changes). In the following sections, we will presents the statistical analysis results we have obtained by studying the BGP raw data collected in 2001/02 at Mae-East and Mae-West, the two major exchange points in North America, which currently host over 60 service providers, including MCI, SPRINT, BBN, ANS etc.

\subsection{1 Änalysis of Gross Trends}

A previous study of the raw BGP data from 1996-1998 showed that since the end of 1997, a gradual rise in the number of announcements was seen [Labovitz99]. Our results obtained by studying the data through 2001, show that the overall trend of BGP traffic, both Announcement and Withdrawal seems to be decreasing (see Figure 3.7 and Figure 3.8). For example, the average number of Announcement for the first few months (Jan, Feb, May, July, Aug, Sept) is 15,711,239 /month, but for the last a few months (Oct, Nov and Dec) is only $3,887,126 /$ month. At the beginning of the chapter, we listed a number of causes that could explain the decrease of the BGP traffic over time. However, we think that the economic downturn in the Telecom sector may be an additional factor. Many 
Telecom businesses, such as some Internet Service providers have been closed in 2001, which would decrease the number of BGP peers, and could definitely contribute to the decline of the BGP traffic. Also, even through in 2001, the overall trend seems to be a decrease in traffic, we can still see some peaks in particular months. For example, in July and September, the volume of the traffic is relatively higher than the adjacent months. It is believed that this may be the result of the worm storm due to CODE RED II in July and NIMDA in September 2001. The two storms generated a significant volume of BGP routing traffic, and caused a lot of damages. [Cowie01].

As of February 1998, the number of announcements per day at Mae-East surpassed the number of withdrawals for the first time [Labovitz99]. Although we omit the details for brevity, this trend holds true for our study on the data collected in 2001. As shown in Table 3.1, on average, the number of Announcements represents $79.9 \%$ of the total BGP traffic volume, and the number of Withdrawals only $20.1 \%$. We may wonder whether the majority of the inter-domain routing traffic is due to new route announcements. The answer is "not exactly". The reason we have up to $79.9 \%$ of Announcements, is that, on one hand, we do get new route announcements, but on the other hand, many announcements are redundant $(56.19 \%$ in average). These are not reflecting real routing changes, but a pathological routing behavior. We will study this factor in more depth in a later section.

Note: As mentioned before that data presented here does not cover the whole year 2001, due to the fact the trace files for March, April and June are not available. 
2001 Arrival Rate/month

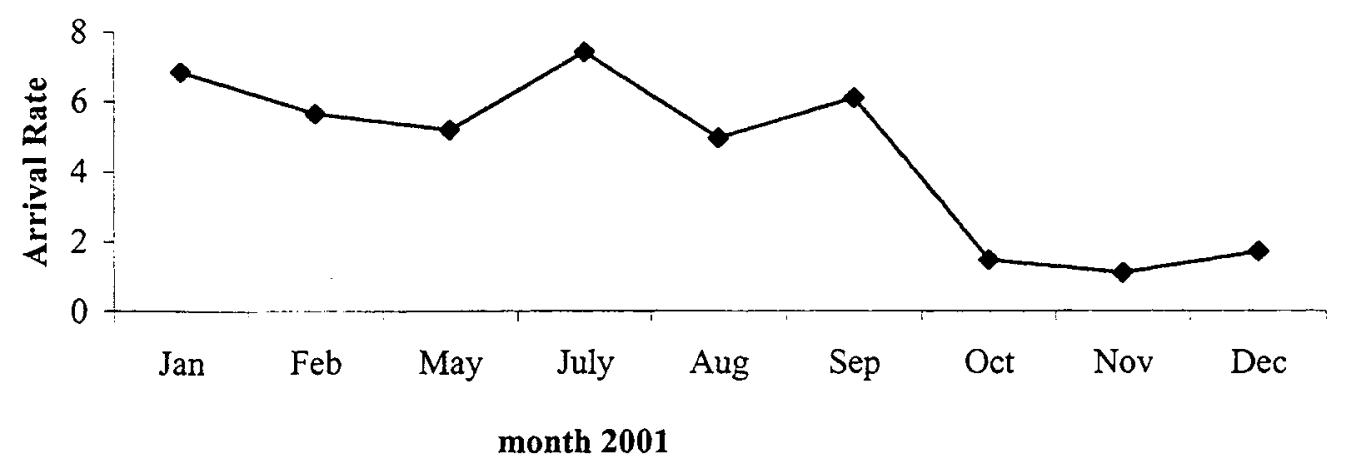

Figure 3.7 Arrival Rate Trend

The mean arrival rate over the nine months period in 2001 is $4.47 / \mathrm{sec}$

BGP Updates (A, W,T) in 2001

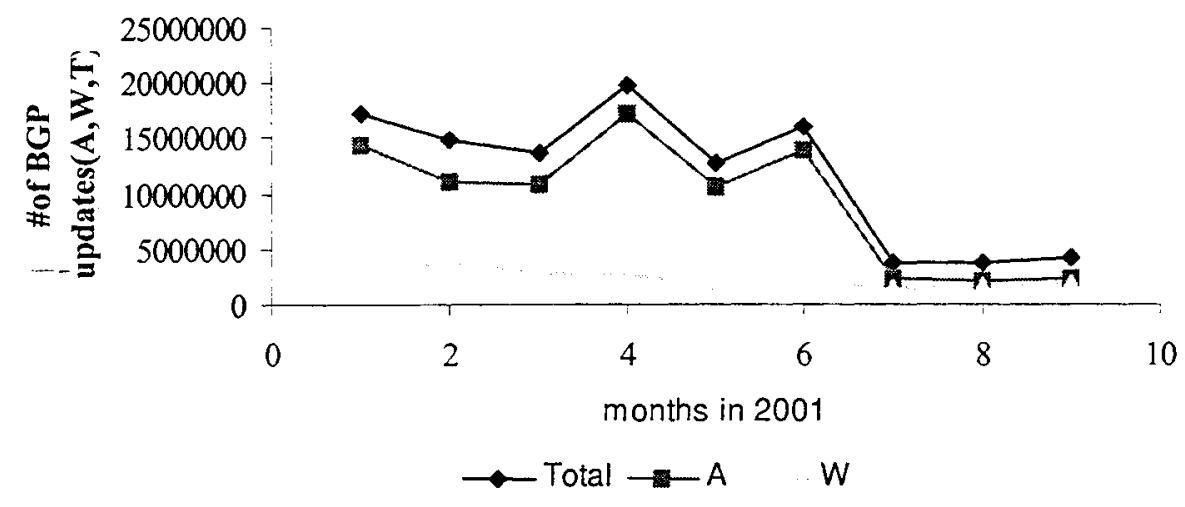

Figure 3.8 BGP Updates Trends

\begin{tabular}{|l|l|l|}
\hline & Mean/month & $\%$ \\
\hline Total & 11792462 & $100 \%$ \\
\hline Announcement & 9422408 & $79.9 \%$ \\
\hline Withdrawal & 2370054 & $20.1 \%$ \\
\hline
\end{tabular}

Table 3.1 Mean of BGP Updates 


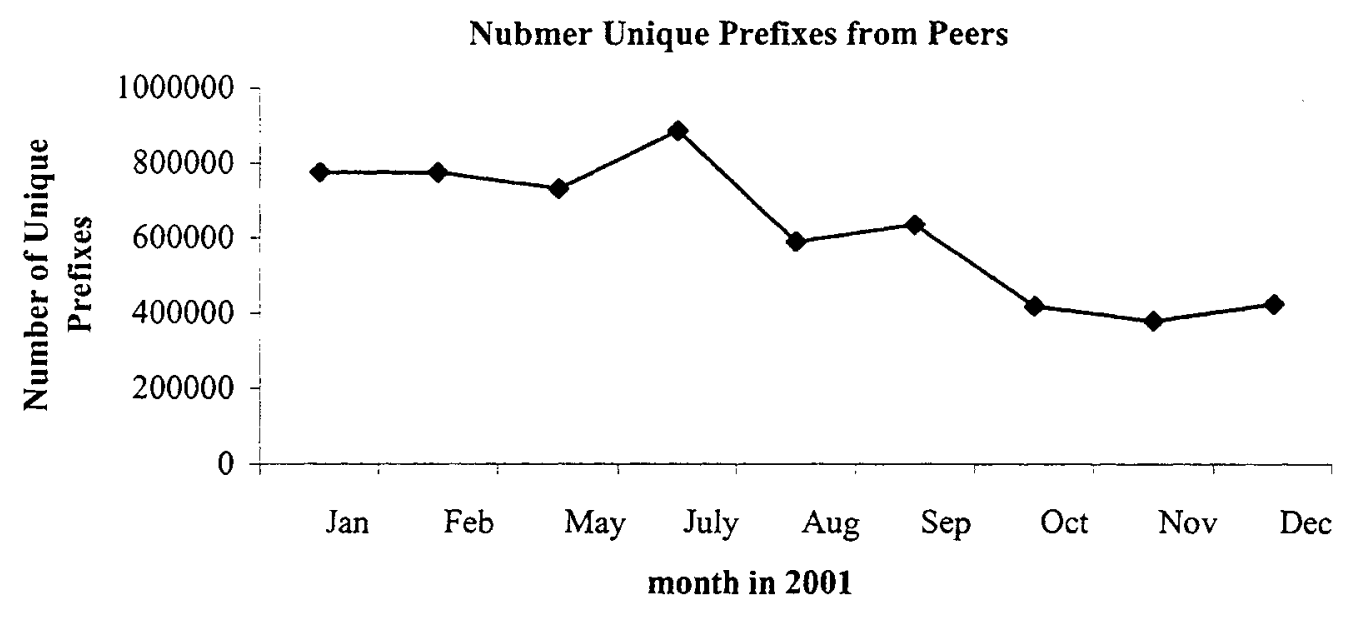

Figure 3.9 Unique Prefixes Trend

Figure 3.10 shows the sum of the number of unique prefixes updated by each peer. It may include redundant counting of the updates on particular prefixes. For example (see Figure 3.10), when PEER_A receives the updates from PEER_B about prefix 210.102.32.0/19, it will count it once. Later on, when it receives the updates about the same prefix from PEER_C, it will count it once more. This is due to the implementation of our analysis "on the fly". In the analysis program, the number of unique prefixes is counted per peer basis, then simply summed up. The intent is not to try to show the exact number of prefixes for the router under testing, but to show the magnitude of the updated prefixes, and then show the trend of the BGP inter-domain traffic. 
210.102.32.0/19

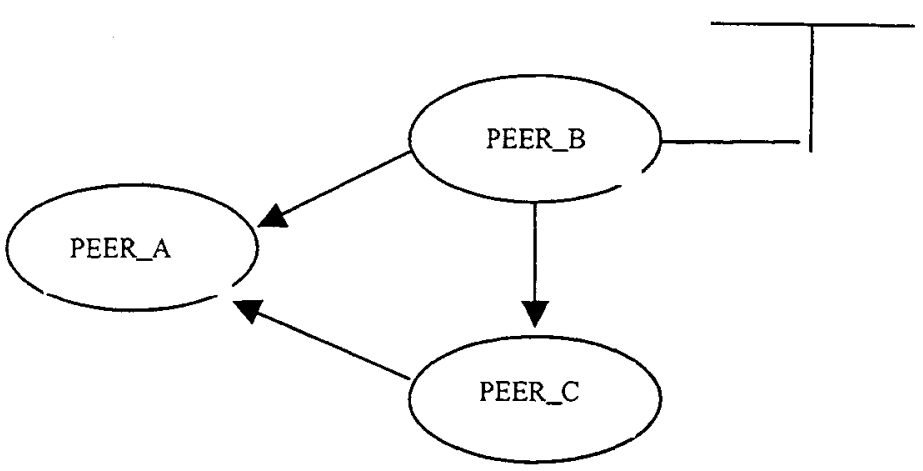

Figure 3.10 Unique prefixes

\subsubsection{Network Livability}

We define Network Livability as the percentage of time when a network (prefix) is up over the prefix's lifetime within the study period (one month).

$$
\text { NetworkLivability }=\text { UpTime/TotalLifeTime*100\% }
$$

where, as shown in Figure 3.11,

UpTime is the sum of the time periods that the prefix is up;

TotalLifeTime is the time period that from the receipt of the first update of a prefix to the end point of the study period.

The reason we define the TotallifeTime from the time when we receive the first update of a prefix, is because the state of the prefix within time $t$ as shown in Figure 3.11 is unknown. Normally, during the study period, if the first update we get is Announcement, we could assume that the prefix's previous state was down; or vice versa, if the first update is Withdrawal, we could assume the prefix was up. However, as the result of our 
study showed that about $56.19 \%$ of the BGP updates are redundant announcements, the high percentage of the redundant updates leave the prefix's state before receiving the first update within the study period uncertain. With our approach, we eliminate the impact of the redundant routing updates, which do not reflect a real network topology change.

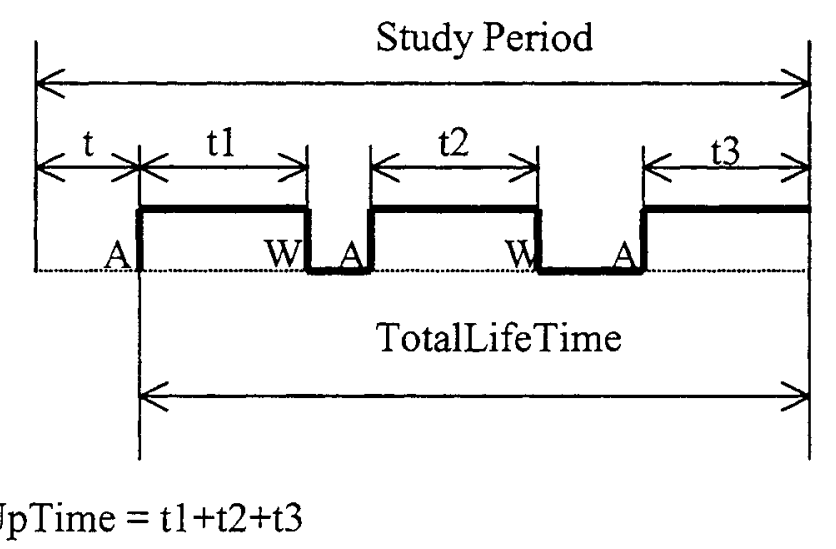

Figure 3.11 Network UpTime

By studying the prefixes over 9 months in 2001, we divided the prefixes into 10 clusters according to their livability rate for each individual month so that we could have a view of how the prefixes are distributed in terms of livability. Given that results for different months show similarity in terms of the livability distribution, here we show only the histograms for January (Figure 3.11) and February (Figure 3.12) of 2001. Assuming that a prefix with livability $>70$ is considered stable, we can see from Figure 3.12 that in January, 2001 about $81.64 \%$, and in February about $82.89 \%$ of prefixes are quite stable. From this result, we can conclude that most prefixes are relatively stable, and the interdomain routing instability mostly stems from a small percentage of prefixes. 


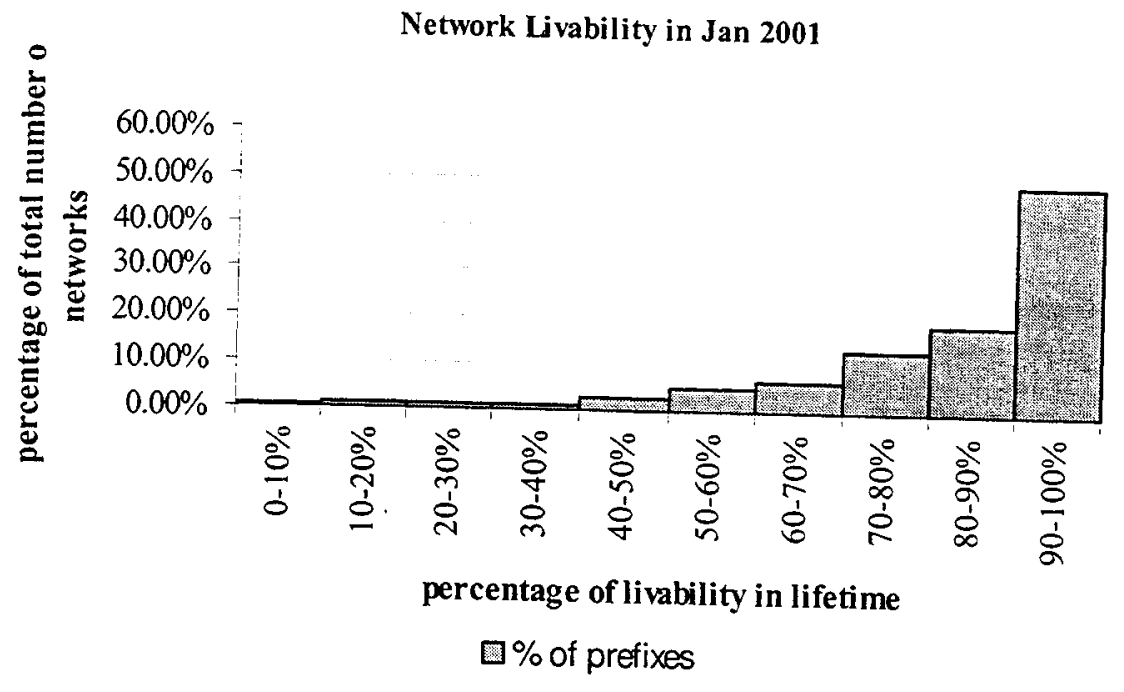

Figure 3.12 Network Livability in January

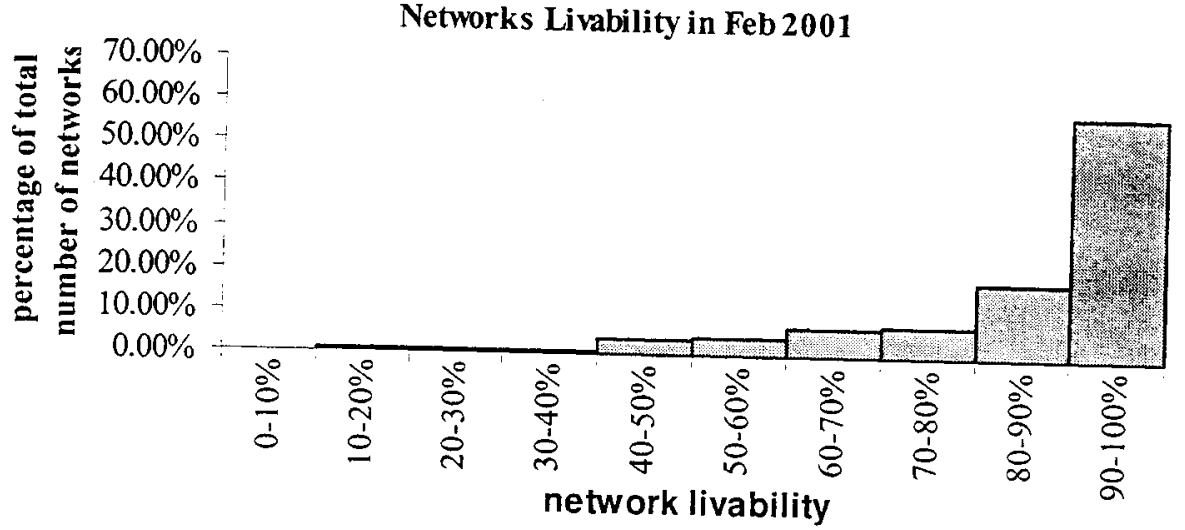

Network Livability

Figure 3.13 Network Livability in February 


\subsubsection{Prefix Length Distribution}

Prefix-Length Distribution Mae-East (2002 Jan)

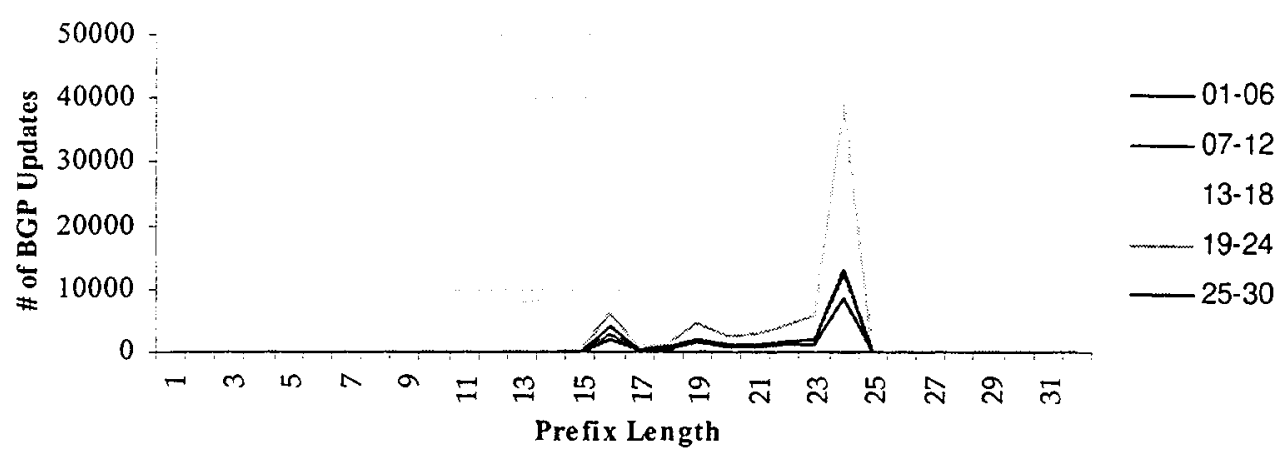

Figure 3.14 Prefix Length Distribution

A prefix represents a set of destination IP address blocks. The length, or network mask, of a-prefix represents the number of possible subnet addresses reachable via that network address. As we mentioned in the Chapter 2, the introduction of classless inter-domain routing (CIDR) has allowed backbone operators to group large numbers of customer network IP addressed into one or more large "supernet" route advertisements at their autonomous system's boundaries. In other words, CIDR got rid of the idea of an implicit mask based on the class of the network; instead, it requires that explicit masks be passed in inter domain routing protocols. CIDR allows network prefixes to be of arbitrary lengths (as opposed to the classful system, in which prefixes had to be even multiple of an octet. [Stewart99]. A prefix may be as specific as a single machine (32 bits), or as general as a default route ( 0 bit); However, in practice, from the prefix length distribution in Figure 3.14, we can observe that aggregate prefix length from 16 to 24 bits are most 
commonly used in inter-domain routing, and the length of 24 is the most often used. This can give us an idea what are the sizes of the prefixes in the real world Internet. Also, it can provide guidance on the value of the network size parameter required by the BGPSim configuration.

\subsubsection{Redundancy}

We measure the redundancy of the BGP updates by the percentage of total number of redundant updates (Announcement and Withdrawal) comparing with the total umber of updates (Announcement and Withdrawal). A redundant Announcement, is a subsequent route announcement that does not differ in any BGP path attribute information from a previous one. A redundant Withdrawal is the repeated transmission of BGP withdrawals for a prefix that is currently unreachable. [Labovitz97]

From Figure 3.15 below, we can see over the 9 months study period, there is still relatively a high percentage of redundant routing information with an average of $56.19 \%$ /month. Comparing with the result from [Labovitz99] that stated that the majority (more than $99 \%$ ) of the routing information was pathological or redundant and did not reflect a real network topology change, the stability of Internet has improved considerably in the last couple of years. However, the amount of pathological updates is still very high, and it continues to contribute to the huge volume of Internet control traffic, and so to network congestion, and waste of network resources. 


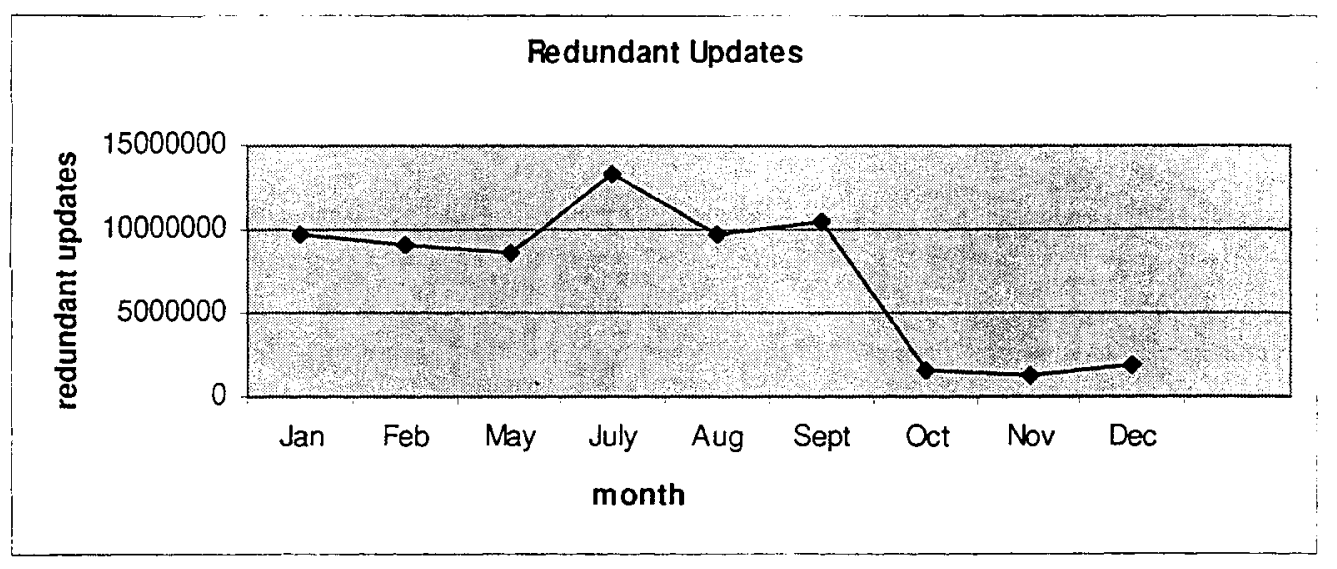

Figure 3.15 Redundant Updates

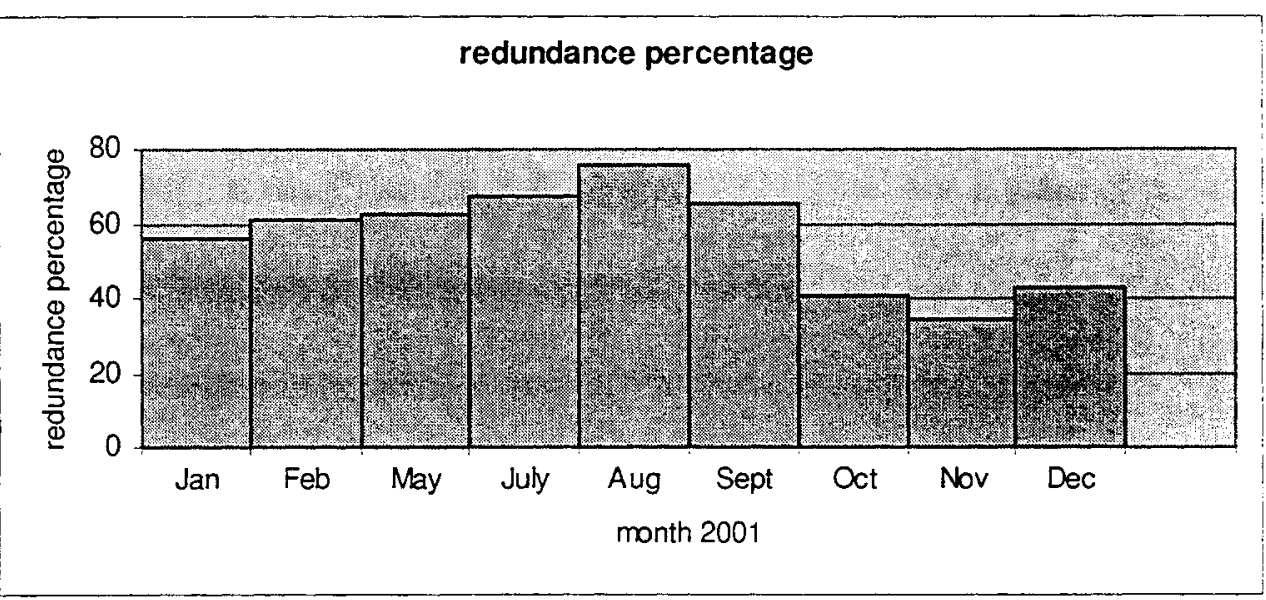

Figure 3.16 Redundant Percentages

Histogram of Redundancy Jan 2002

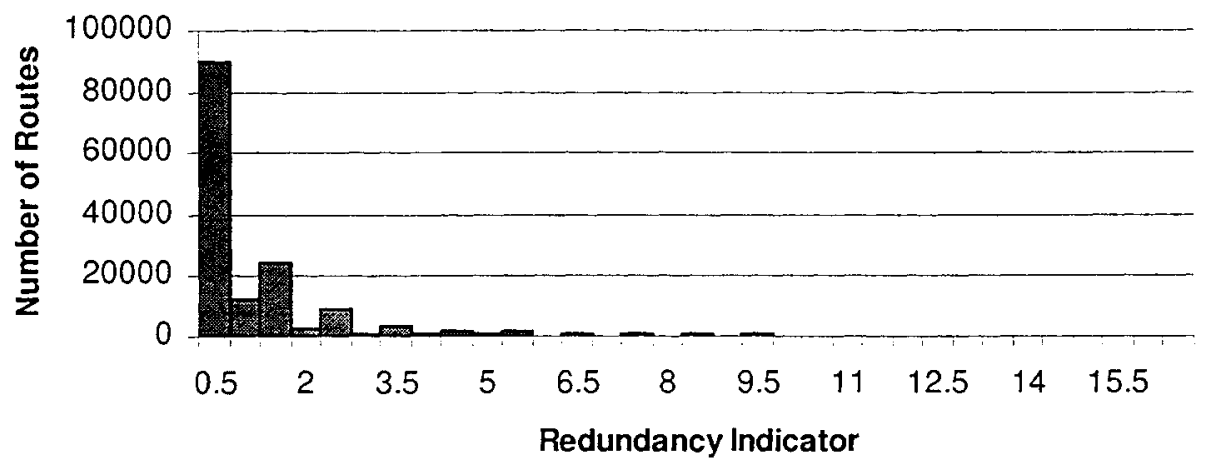

Figure 3.17 Redundancy Indicator in Jan 
Ideally, the BGP updates should only be generated when there is a policy change or topology change, and the number of updates on a particular prefix should reflect the stability of the prefix. One may wonder whether the redundant updates come from only some prefixes or are common for all the prefixes? This is why we defined the Redundancy Indicator as follows:

We define the Redundancy Indicator as following:

UpRedundIndicator = \#RedundUPupdates / \#StateChangeToup

DownRedundIndicator = \#RedundDownUpdates / \#StatechangeToDown

Ideally, there should not be any redundant updates, thus the Redundancy Indicator should be zero. But in our study, within a total of 151,938 routes in January 2002, we obtained:

... UpRedundIndicator:

Max value: there are only 2 routes with UpRedundIndicator between $70.0-70.5$

Min value: 0

Mean value: 1.738

DownRedundIndicator:

Max value: 0

Min value: 0

Mean value: 0 
Since DownRedundIndicator is 0 , this means that all the redundant updates are in fact redundant announcements. Considering the redundancy, this is a complete reversal from the study result in [Labovitz97] and [Labovitz99], which stated that the majority of BGP updates in 1997 , about $99 \%$, consisted entirely of pathological, duplicate withdrawals. We can conclude that the BGP traffic characteristics have changed dramatically since then. As we observed from our previous results that only about $20.1 \%$ of the BGP traffic over 2001 period are withdrawals, and none of them is redundant. In [Labovitz97], it was presumed that the possible causes for the high percentage of redundant Withdrawals in 1997 could be the stateless BGP implementation by some vendors, which means:

- no state maintained on what is advertised to peers.

- when receiving any change, transmit withdrawal to all peers regardless of whether previously notified or not.

- send updates for both explicit and implicit withdrawals.

By 1998 , most vendors had BGP implementations with partial state, and, the number of redundant Withdrawals was reduced by an order of magnitude. By 2001, we found in our study, that there are no more redundant Withdrawals, which is a considerable improvement.

\subsubsection{State Change Per Route}

One thing would be interesting to mention how many updates reflect an actual state change that a given route is going up or down. This can be regarded as an indicator of the inter-domain routing instability for a route. 
In this analysis, we studied more recent data collected at Mae-West exchange point in January 2002. We divided the data into 5 equal periods of 6 days each, except the last period is 7 days, and tried to see if there is a pattern in the distribution of state changes per route. As we can observe from the Figures $3.18,3.19,3.20,3.21$, and 3.22 below, it shows a similarity among the different periods: few routes suffer a large number of state changes, whereas the majority of routes have a small number of state changes. In other words, the majority of routes are relatively stable, and only a small percentage of the routes contribute the most to the instability of inter-domain routing.

State Change (01-06, 2002 Jan)

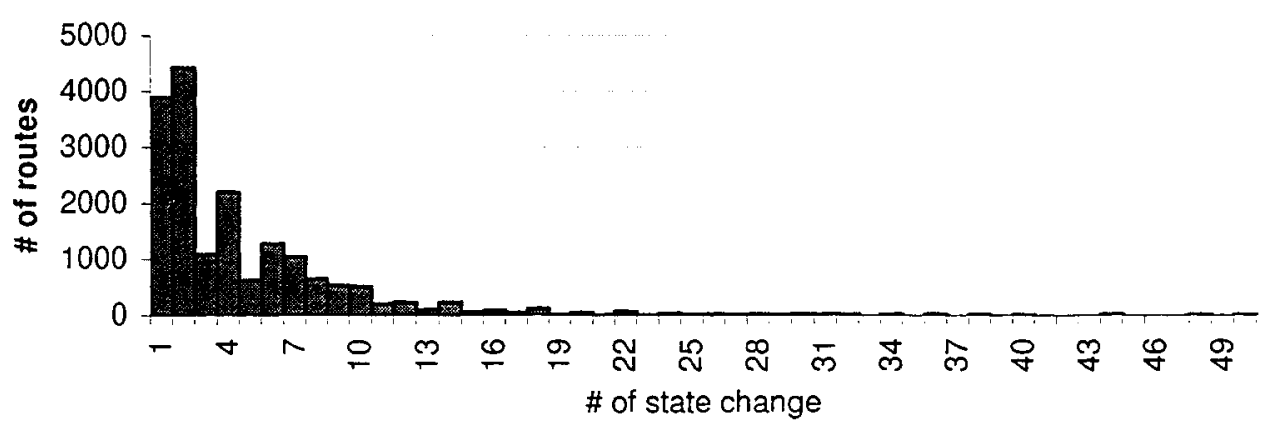

Figure 3.18 State Change (01-06 Jan, 2002)

stateChange (07-12,2002 Jan)

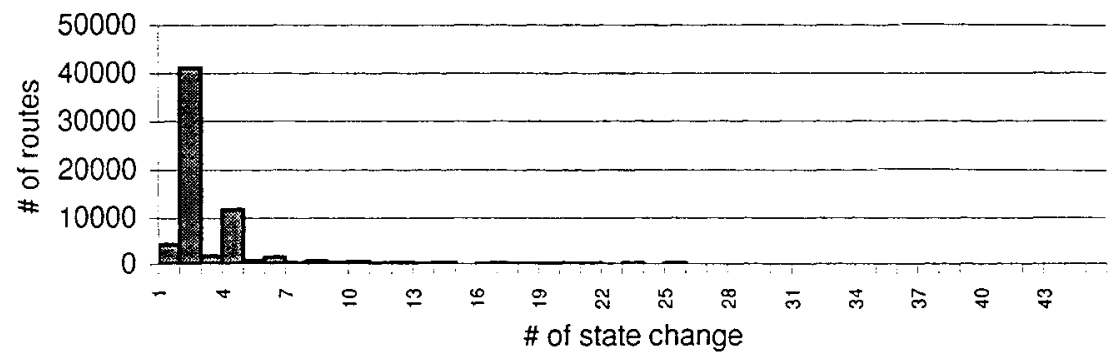

Figure 3.19 State Change (07-12 Jan. 2002) 


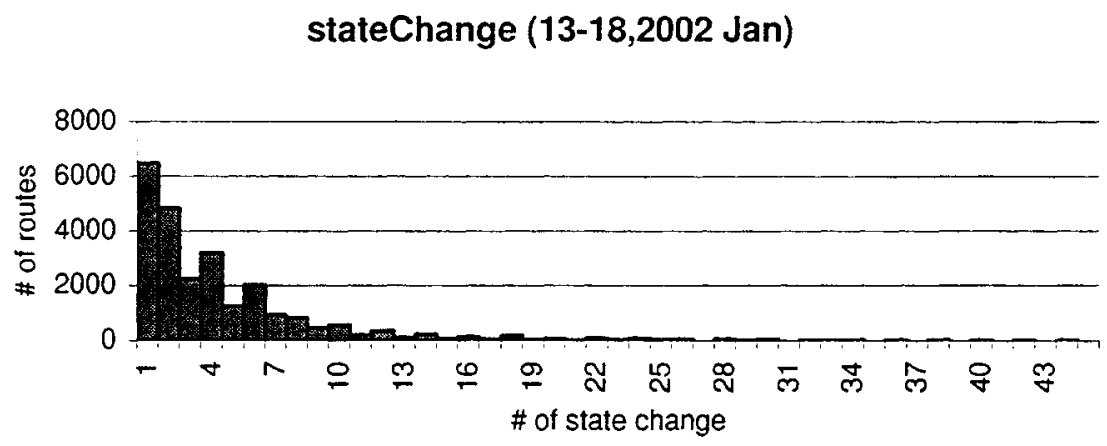

Figure 3.20 State Change (13-18 Jan, 2002)

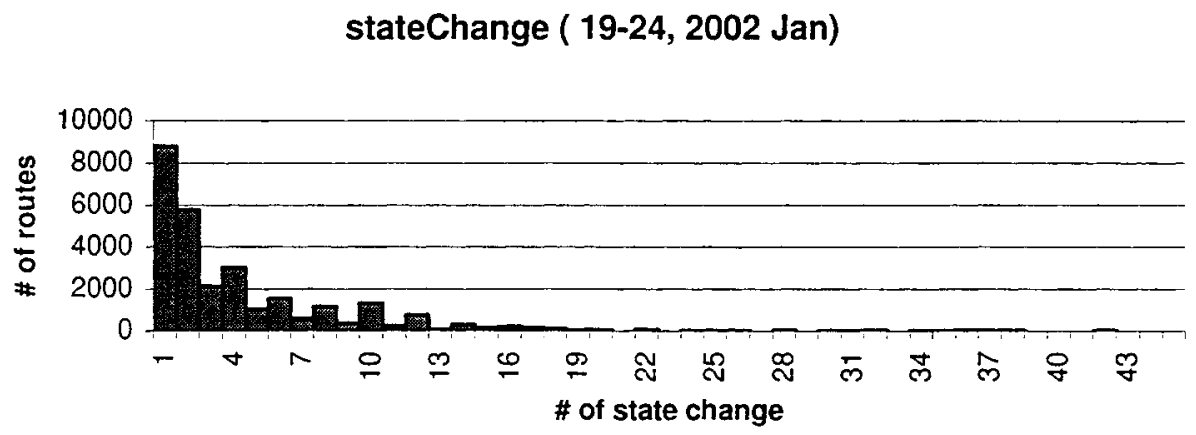

Figure 3.21 State Change (19-24 Jan, 2002)

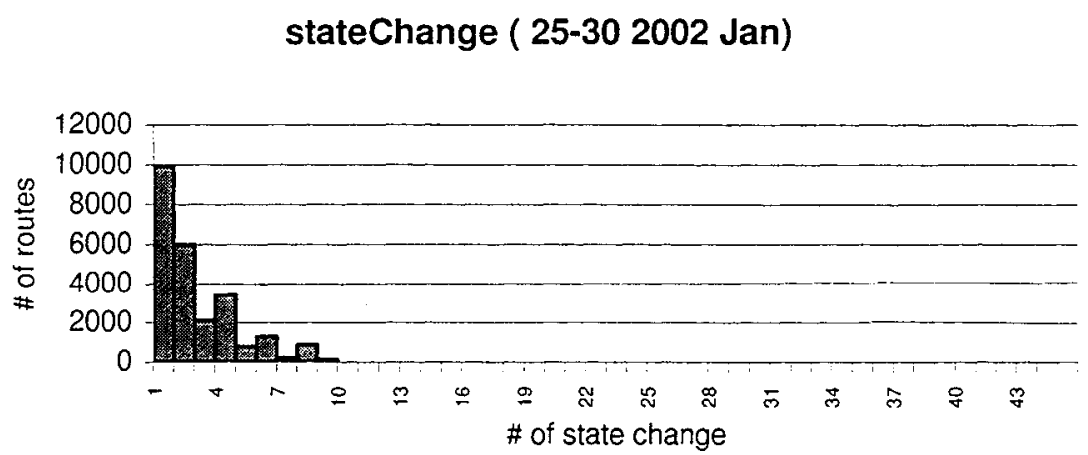

Figure 3.22 State Change (25-31 Jan, 2002) 


\subsubsection{Weekly and Daily Pattern}

It is interesting that we can observe weekly and daily pattern in the BGP traffic. The magnitude of routing information exhibits the same significant weekly and daily cycles as the network usage and congestion.

Weekly Pattern in 2002 Jan

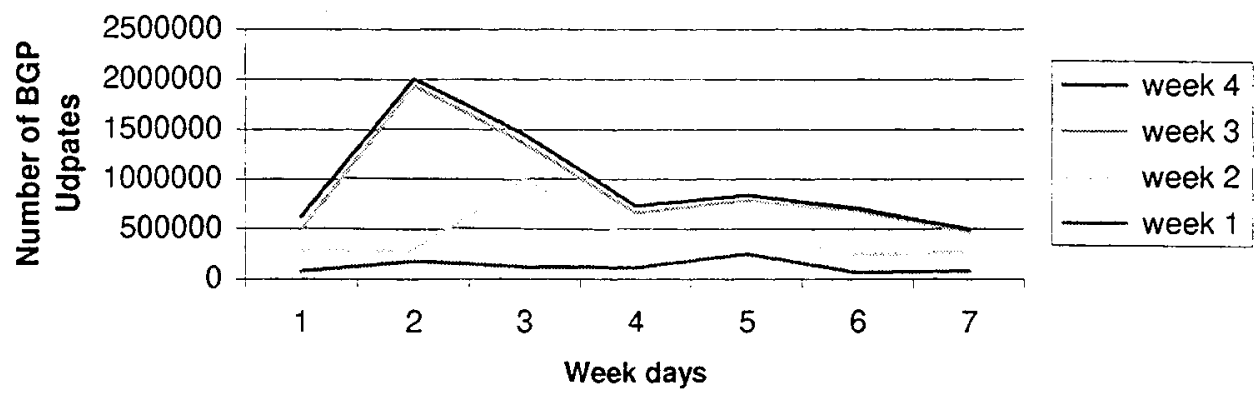

Figure 3.23 Weekly Pattern

Days Pattern 2002 Jan

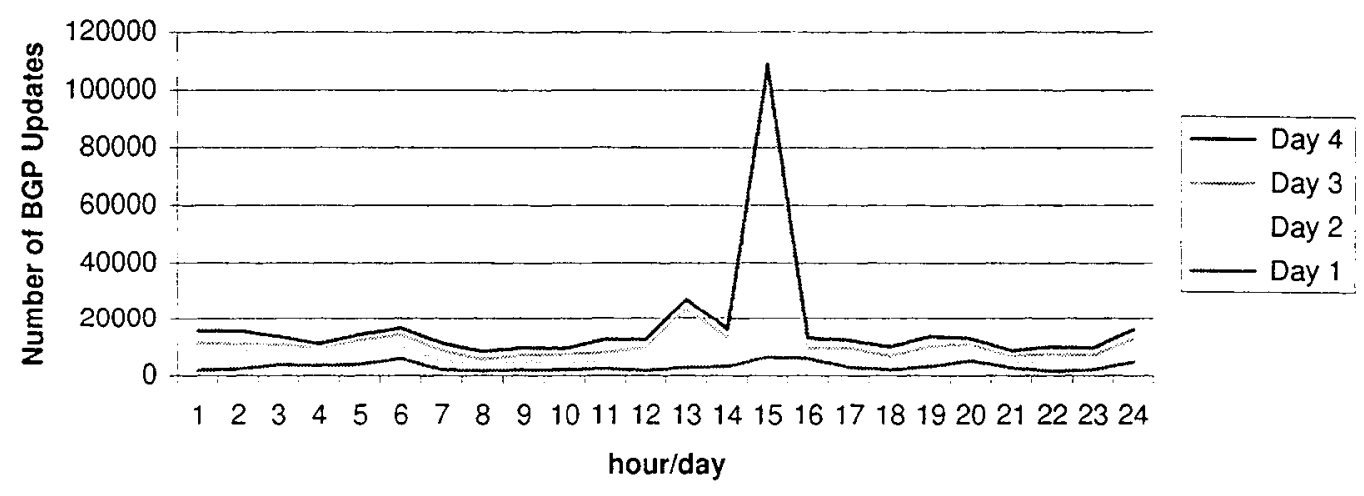

Figure 3.24 Daily Pattern 
From Figure 3.23, we can observe that the volume of the BGP traffic tends to be low at weekends and high on workdays, which indicates a strong correlation between the level of routing activity and network usage.

From Figure 3.24, we can notice that the updates seem to have a peak value in the afternoon from 1:00pm to $3: 00 \mathrm{pm}$. For the rest of the hours, there are some small peaks, but are relatively evenly distributed.

The reason we see a correlation between the BGP routing information and the network usage is because at high load, some links of the networks could be congested, and can trigger some routing control traffic loss, or even bring down the BGP peer sessions. This can lead to the loss of the connectivity, introduce the routing instability, and increase the volume of the routing traffic.

\subsection{K-means Clustering Analysis}

To be useful in performance evaluation, analytical models requires classes of homogenous components with respect to the resource usage. Real workloads can be viewed as a collection of heterogeneous components. And because of the heterogeneity, the representation of a workload by a single class often lacks accuracy. By partitioning the workload, we can improve the representativeness of the characterization and increase the predictive power of a performance/simulation model. Partitioning techniques divide the workload into groups of homogeneous components. The aim is to group components that are somehow similar to each other. But what attributes should be used as a basis for 
the measure of similarity? The BGP updates, Withdrawals and Announcements, reflect legitimate policy or forwarding changes, thus represent to a certain degree the availability or stability of a route. The number of announcements and withdrawals for a particular route can be regarded as two critical attributes of a route. But how should we determine the groups of routes with similar updates patterns? The answer lies in clustering. In our study, we applied the $k$-means clustering technique to process, and we were able to group the routes with respect to some properties of the updates. In the following section, we will discuss briefly the clustering algorithms we implemented, then show the results obtained from clustering analysis.

\subsubsection{Standard Deviation}

In our clustering analysis, we use standard deviation, traditionally denoted by $\sigma$, which is defined as the square root of the variance, to measure the spread of the distribution. The quantity $(x-\mu)^{2}$ represents the square of the distance between $x$ and its mean $\mu$. The expected value of this quantity is called $\operatorname{Var}(\mathrm{x})$ [Jain91]:

$\operatorname{Var}(x)=\sigma^{2}=E\left[(x-\mu)^{2}\right]=\sum p_{i}\left(x_{i}-\mu\right)^{2}=\int\left(x_{i}-\mu\right)^{2} f(x) d x$

\subsubsection{The k-means Algorithm}

The goal of the $\mathrm{k}$-means clustering is to allocate the points into $\mathrm{k}$ clusters such that the intra-cluster variance is minimized while the inter-cluster variance is maximized. The following is the k-means algorithm we implemented. It starts by making an initial guess on the number of cluster $\mathrm{k}$, then find $\mathrm{k}$ points to be initial estimates of centroids for the $\mathrm{k}$ 
clusters. Next, it examines each point and assigns it to one of the clusters based on the minimum distance. Then it repeats the same procedure until all the points are grouped into the final required number of clusters. The steps of the k-means algorithm are shown in Figure 3.25 [Sisu].

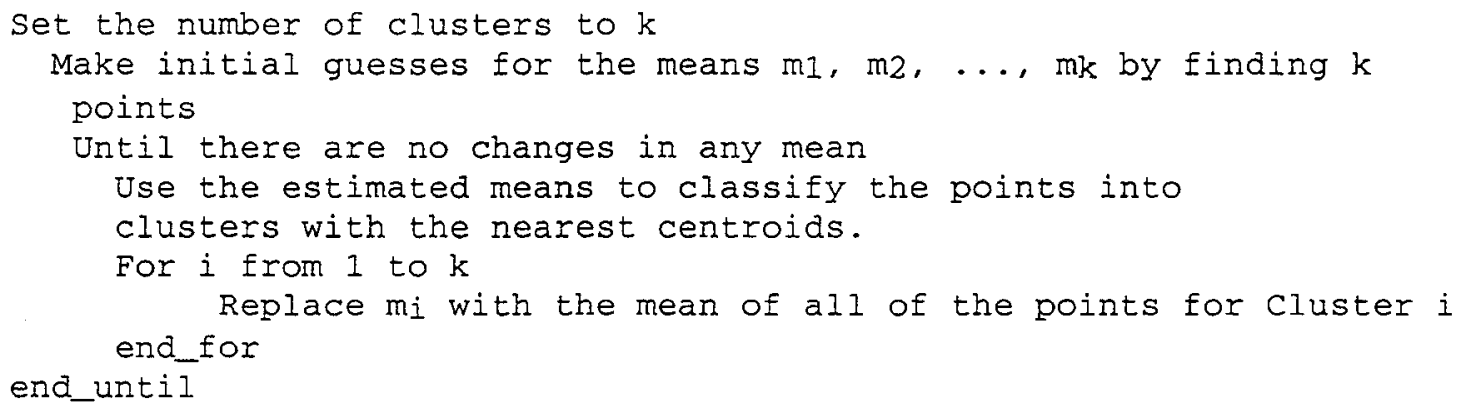

Figure $3.25 \mathrm{k}$-means clustering algorithm

A common problem found in clustering analysis is the difficulty of deciding the number of clusters present in the data. The rule of thumb for clustering is to minimize the intracluster variance while maximizing the inter-cluster variance. [Jain91] Thus in theory, we could achieve this goal by making the number of clusters equal to the number of the points. However, this is not a satisfactory solution because we want a compact representation of the data. Therefore, we have to choose a relative small number of clusters with small intra-cluster variance but large inter-cluster variance. Here is the algorithm we used to determine the quality of clustering [Menasce99]:

Step 1: Calculate the mean of the points in each cluster.

$$
\bar{Y}=\frac{1}{m} \sum_{i=1}^{m} Y i
$$


where $m$ is the number of points in a cluster.

Step 2: Calculate the inter cluster distances.

Step 3: Calculate the mean of inter cluster distances.

Step 4: Find out the standard deviation of mean inter cluster distance (see 5-4).

Step 5: Find out the mean of the Intra cluster distance.

Step6: Calculate the mean of mean intra-cluster distances

Step7: Calculate the standard deviation of mean intra-cluster distances

Step 6: Find the coefficient of variation of mean intra cluster distance

$$
\mathrm{CV}=\sigma / \mu
$$

Step 8: Compute $\beta_{\mathrm{cv}}$ as the ratio of covariance of mean intra cluster distance to covariance of mean inter cluster distance

Step 9: Repeat the procedure for various values of $\mathrm{k}$.

Step 10: Plot this for different value of the number of clusters. Choose the number of clusters after which this value doesn't fall much.

\subsubsection{Clustering Analysis Results}

The following clustering analysis is based on the data collected from the Mae-West exchange point in 2002 January. As we already described in 4.3 .5 that within certain amount of time, the number of state changes can represent the degree of the route stability, we use k-means clustering technique to analyze the data to see if there are homogeneous groups among the routes with respect to the stability of a route. As we 
expected, the result turns out that the routes are shown in groups of similarity in terms of the route stability.

\section{Quality of Clustering}

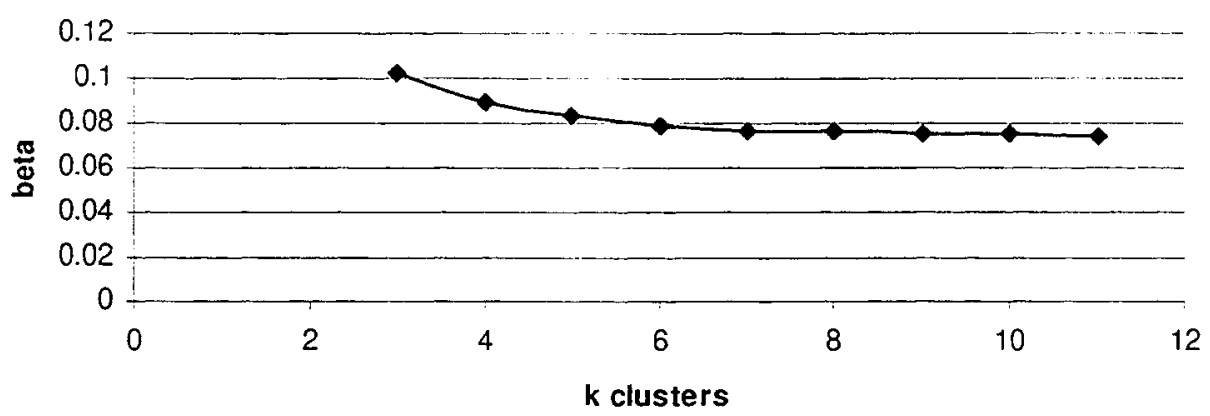

Figure 3.26 Quality of Clustering

From 3.4.3, we can get the data that how many routes correspond to how many actual state changes for each 6 days in January 2002. We calculate the mean of the five 6 -day periods in terms of how many routes correspond to how many actual state changes. Then we applied the k-means algorithm to this set of data. There are total of 119,887 points. Each point represents how many actual state changes occurred during a 6 days period corresponding to a particular route. By applying the algorithm to determine the number of clusters, we obtain the graph shown in Figure 3.26, which gives the $\beta_{\mathrm{cv}}$ value corresponding to different $\mathrm{k}$ values. The point $\mathrm{k}=8$ is chosen as the proper value for $\mathrm{k}$. The mean for each cluster is listed in the following table:

\begin{tabular}{|l|l|l|l|l|l|l|l|l|}
\hline $\mathrm{K}=$ & 1 & 2 & 3 & 4 & 5 & 6 & 7 & 8 \\
\hline Mean \# of state change/6 days & 13 & 2.5 & 24.5 & 36 & 6.5 & 46.5 & 65 & 74 \\
\hline Stability(sec) & 39876 & 207360 & 21159 & 14400 & 79753 & 11148 & 7975 & 7005 \\
\hline
\end{tabular}

Table 3.2 Route Stability Clustering 
In Chapter 2, we mentioned that one of the problems when using BGPSim was that there is no guidance on what value to define for "stability" in the BGPSim configuration file. With this clustering analysis result, we can define groups of network-lists in the BGPSim, so that each group will represent routes at different levels of stability according to Table 3.2. This way we can represent more accurately the characteristics of the real-world routing traffic.

\subsection{Summary}

In this chapter, we have characterized the BGP routing traffic on the data collected through 2001 and Jan of 2002, and here is the summary of our findings:

1. From 1996 to 2001, even though the size of the Internet has increased dramatically, there is a considerable drop in the average number of BGP updates per day at MAEEAST exchange router (from 3-5 millions in 1996 to 0.3 millions in 2001). The order of magnitude drop is mainly due to the improvements of the software, and hardware provided by various vendors, and to some new technologies introduced, such as high aggregation, flapping damping, etc.

2. The mean number of BGP updates per month in 2001 was $11,792,462$, out of which 9,422,408 were Announcements and 2,370,054 Withdrawals. 
3. For the 9 months studied in 2001 , the mean arrival rate of the BGP updates is $4.47 / \mathrm{sec}$.

4. From our study, we can still observe a high percentage of update redundancy. Over the 9 months study period, $56.19 \%$ of updates are redundant. However, this is much better than $99 \%$ redundant updates reported in [Labovitz97].

5. We defined two Redundancy Indicators, which gives the percentage of redundant updates (Announcements and Withdrawals). Surprisingly, the DownRedundIndicator is 0 , which means that there are no redundant Withdrawal messages and all of the redundant updates are Announcements. This represent an important change compared to the result from [Labovitz97] where the majority of the redundant updates were Withdrawals. The mean Up Redundancy Indicator found for 2001 is 1.738.

6. The trend in terms of the number of the BGP routing information updates is continuously decreasing in 2001, which matches the result from [Labovitz99]

7. By introducing the concept of Network Livability, which shows the fraction of time a network (prefix) is up over the prefix's lifetime within the study period, we can see that more than $80 \%$ of prefixes (networks) were up over $70 \%$ of the time in 2001 . From this result, we can conclude that most prefixes are relatively stable, and the inter-domain routing instability stems mostly from a small percentage of prefixes. 
However, we are not sure if the cause is the originating networks itself or some unstable nodes along the route.

8. The BGP traffic does show weekly and daily patterns. The magnitude of BGP traffic exhibits the same significant weekly, daily patterns as network usage and congestion, which was also stated in [Labovitz99]. However, we gave more data to support this conclusion.

9. The majority of routes are relatively stable, and only a small percentage of the routes are contributing the most to the instability of inter-domain routing.

10. We used k-means clustering analysis technique to analyze the stability of the prefixes and provide guidance for the value of the network stability, which can be used to construct a configuration file for BGPSim.

11. We can observe that aggregate prefix lengths from 16 to 24 bits are commonly used in inter-domain routing, and the length of 24 is the most often used. This can give us an idea what are the sizes of the prefixes in the real world Internet. 


\section{CHAPTER 4. MEASUREMENT OF BGP UPDATE PROCESSING}

Generally, in Internet, the BGP protocol takes several minutes to reach a consistent view of the network topology after a route change. These delays stem from temporary routing table oscillations that are formed during the operation of the BGP path selection process on the routers. During these periods of delayed convergence, end-to end Internet paths will experience intermittent loss of connectivity, as well as increased packet loss and latency, so that introduce instability to the Internet [Labovitz97]. Thus, it is important to understand what factors have an impact on the Inter-domain routing convergence. An important factor is the speed of processing routing update messages by a BGP router. In this section, we present the results derived from our experimental measurements of BGP update processing.

\subsection{Experiments Configuration}

The experiment configuration is as shown in Figure 4.1. We configured a network with 3 E-BGP peers; one BGPSim and two commercial BGP routing processes, on 3 separate SUN workstations. The commercial BGP routing process on workstation 2 is our study component, and from now on we will use the term, TestingNode, to denote it. The BGP routing process on workstation 3 serves as a peer to the TestingNode. In the experiments, we use BGPSim to generate BGP traffic and to inject it to the TestingNode. On workstation2, we use the Quantify performance tool to measure the TotalProcessingTime, which is defined as the time period from the 
receipt of the first $\mathrm{BGP}$ announcement to the time it completes the processing of the updates.

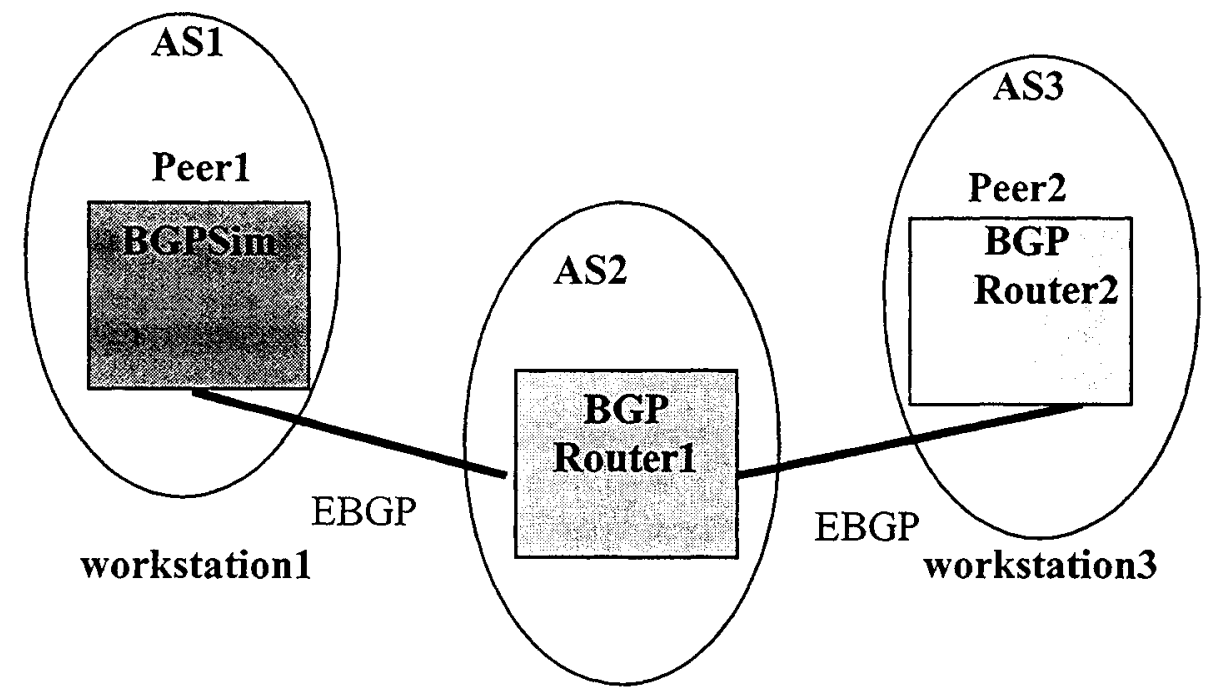

workstation2

Figure 4.1 Experiment Configuration

\subsection{BGPSim Configuration File Generation Scripts}

In order to make the BGPSim configuration file reusable and to save the effort of creating different configuration files by hand we wrote an Expect script, which can construct automatically different configuration files based on the user's input. The User will be prompted to enter the BGPSim and its peer AS numbers, the IP addresses, how many routes will be included in one update message, and how many routes to be advertised to the peer from BGPSim. After the user enters the required information, the script will automatically generate a "sample.conf" file, which will be used as the BGPSim configuration file. An issue we want to highlight here is that 
currently most vendors' routing table implementations are in a tree structure. If the tree is created unbalanced, this will affect the average search time and may have an impact on the results of the experiment. Therefore, in order to generate a synthetic workload that exhibits more accurately the characteristics of the real-world traffic, it is important to use IP addresses that are evenly distributed in the tree structure. In addition, we use in the Expect script the results obtained from the clustering analysis shown in Table 3.2, to classify the networks into 8 categories. Each category represents a different level of network stability. Based on those considerations, the generated BGPSim configuration will have eight categories of advertised IP addresses carefully chosen to provide a balanced tree structure. The routes are randomly distributed in these eight categories, and each routing update will have different path attributes.

Below is an example of the usage of the Expect script:

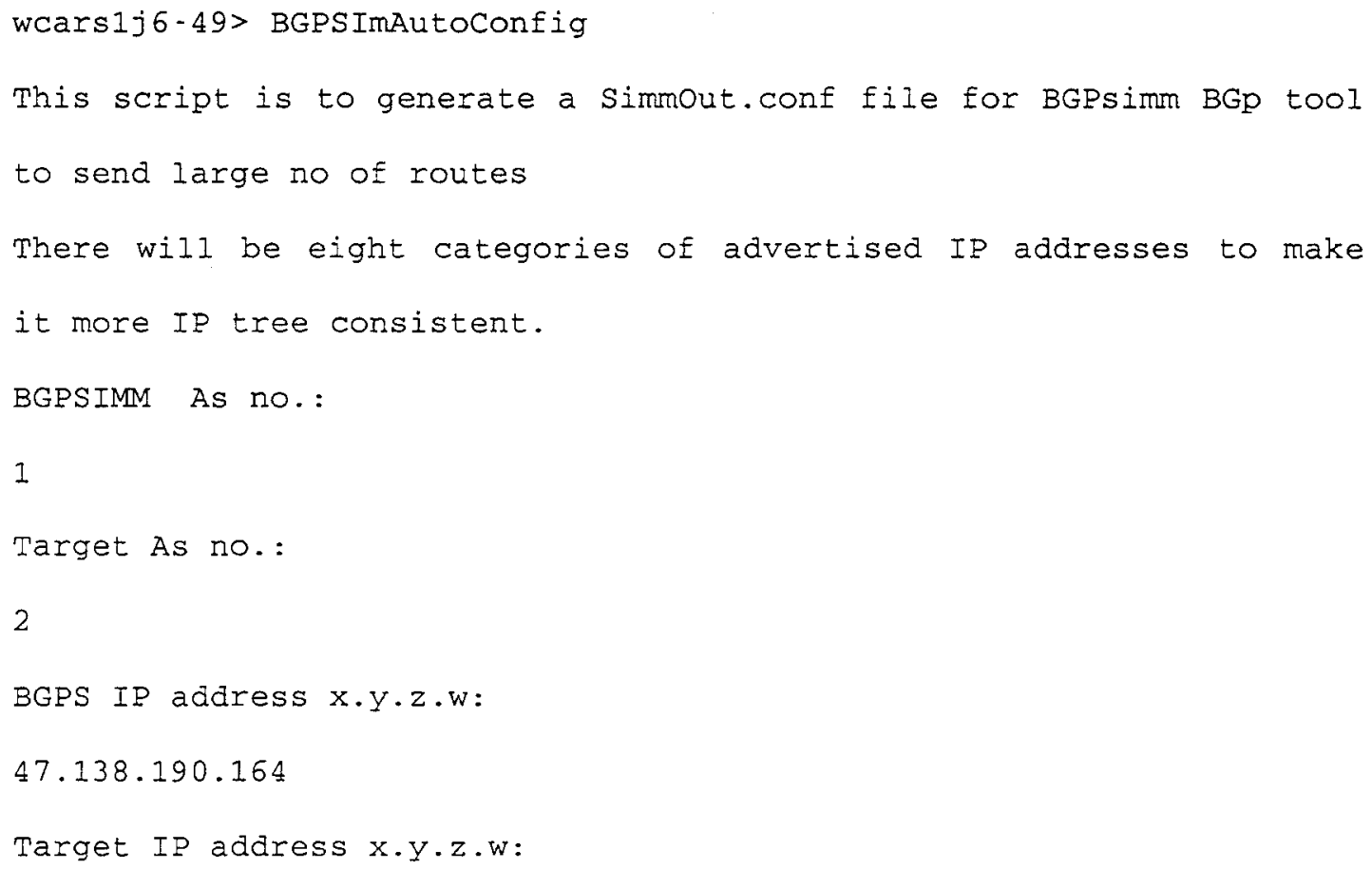


$45.128 \cdot 185.214$

No. of routes per update(Iess than 250):

20

No. of routes:

10000

. . etc.

\subsection{Study of the impact of the policy on TotalProcessingTime}

\subsubsection{Experiment 1}

Characteristics of the experiment:

- we announce 25091 routes (TotalRoutes) through BGPSim to the TestingNode.

- set simple AS import policies to reject $0.00 \%, 39.80 \%, 60.20 \%, 80.61 \%$ of the

TotalRoutes respectively.

- no export policy.

The results are shown in Table 4.1 and Figure 4.2.

\begin{tabular}{|l|l|l|l|l|l|}
\hline Accepted & Rejected & \%rejected & START & STOP & DELAY \\
\hline 25,091 & 0 & $0.00 \%$ & $14: 32: 59$ & $14: 34: 35$ & $0: 01: 36$ \\
\hline 15,106 & 9,985 & $39.80 \%$ & $16: 30: 39$ & $16: 33: 59$ & $0: 03: 09$ \\
\hline 9,986 & 15,105 & $60.20 \%$ & $16: 42: 46$ & $16: 45: 55$ & $0: 03: 20$ \\
\hline 4,866 & 20,225 & $80.61 \%$ & $10: 24: 42$ & $10: 28: 47$ & $0: 04: 05$ \\
\hline
\end{tabular}

Table 4.1 Impact of Policy on TotalProcessingTime (1) 


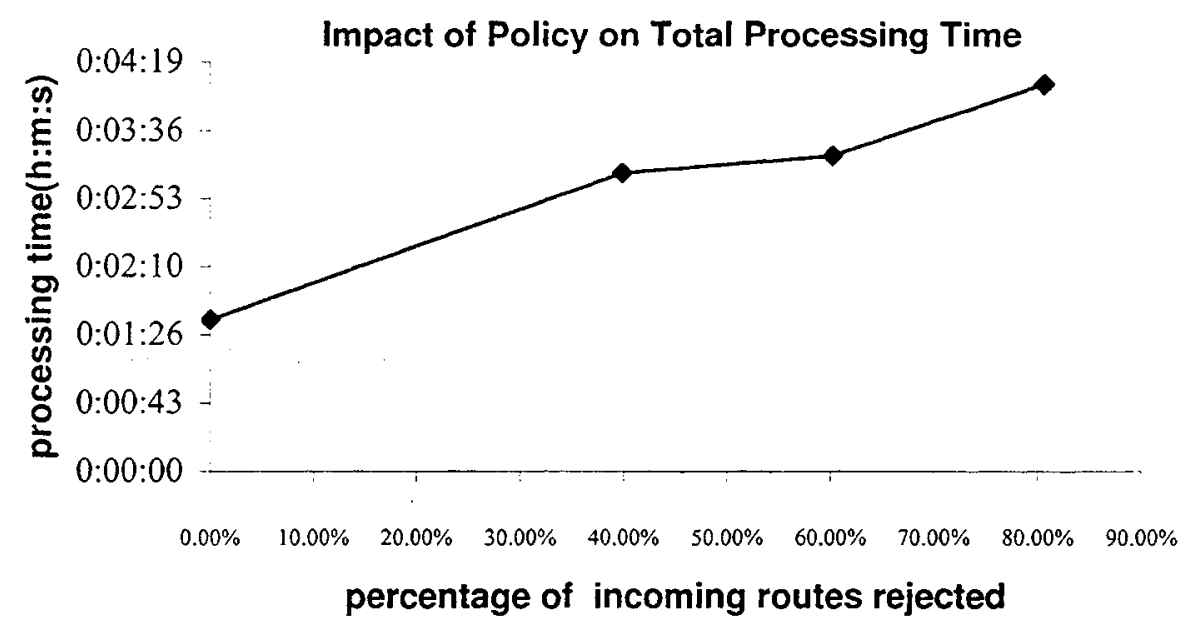

Figure 4.2 Impact of Policy on TotalProcessingTime (1)

\subsubsection{Experiment 2}

Characteristics of the experiment:

- we announce 30211 routes (TotalRoutes) through BGPSim to the TestingNode.

- set simple AS import policies to reject $0.00 \%, 7.63 \%, 16.10 \%, 20.34 \%, 28.80 \%$ of the TotalRoutes respectively.

- no export policy.

The results are shown in Table 4.2 and Figure 4.3:

\begin{tabular}{|l|l|l|l|l|l|l|l|}
\hline Froutes & Accepted & rejected & \%rejected & START & STOP & DELAY & Comments \\
\hline 30,211 & 30,211 & 0 & $0.00 \%$ & $12: 58: 27$ & $13: 00: 22$ & $0: 01: 55$ & \\
\hline 30,211 & 27,906 & 2,305 & $7.63 \%$ & $13: 02: 47$ & $13: 05: 35$ & $0: 02: 48$ & \\
\hline 30,211 & 25,346 & 4,865 & $16.10 \%$ & $13: 45: 46$ & $13: 49: 05$ & $0: 03: 19$ & \\
\hline
\end{tabular}




\begin{tabular}{|l|l|l|l|l|l|l|l|}
\hline 30,211 & 24,066 & 6,145 & $20.34 \%$ & $14: 00: 07$ & $14: 03: 54$ & $0: 03: 47$ & \\
\hline 30,211 & 22,786 & 8,700 & $28.80 \%$ & & & & Connection to Peer1 \\
\end{tabular}

Table 4.2 Impact of Policy on TotalProcessingTime (2)

\section{Impact of Policy on Total Processing Time}

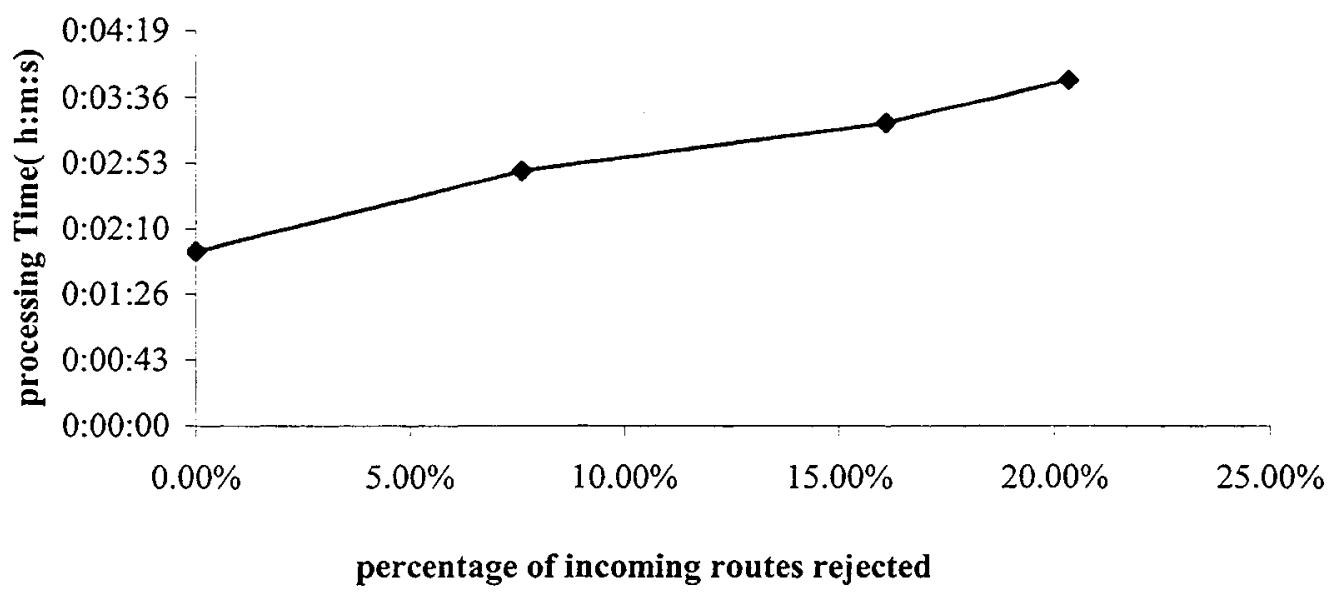

Figure 4.3 Impact of Policy on TotalProcessingTime (2)

Comparing with experiment 1 , in experiment 2 we increase the number of routes injected into the TestingNode to 30211 . The result is that the TestingNode failed to maintain the BGP sessions with its peers when we tried to apply import policy on $28.80 \%$ of the total routes injected. When we look into the implementation details, we found that in this vendor's implementation, all the routes injected are stored in a linked list sorted by IP address. When applying an import policy, a linear search takes place to find the route on which to apply the policy on. It is known that a linear search is expensive, and its complexity grows with the length of the list. This explains why when we increase the total injected routes to 30211 and apply a policy to $28.8 \%$ of the routes, the TestingNode 
cannot maintain the BGP sessions with its peers. The expensive search keeps the CPU too busy to send out the Keepalive messages before the HoldTimer expires. We believe that a better search algorithm could reduce the negative impact on the Total Processing Time, and make the routing component more scalable. In order to verify our hypothesis, we conducted another experiment, which continues to increase the total number of routes injected into the TestingNode while applying import policies on the new learned routes.

\subsubsection{Experiment 3}

Characteristics of the experiment:

- we announce 39427 routes (TotalRoutes) through BGPSim to the TestingNode.

- set simple AS import policies to reject $0.00 \%, 3.25 \%, 18.60 \%$ of the TotalRoutes respectively.

- no export policy

The results are shown in Table 4.3:

\begin{tabular}{|l|l|l|l|l|l|l|l|}
\hline \#routes & Accepted & Rejected & \%rejected & START & STOP & DELAY & Comments \\
\hline 39,427 & 39,427 & 0 & $0.00 \%$ & $11: 55: 24$ & $11: 58: 27$ & $0: 03: 03$ & \\
\hline 39,427 & 38,146 & 1,281 & $3.25 \%$ & $12: 02: 58$ & $12: 06: 27$ & $0: 03: 29$ & \\
\hline 39,427 & 32094 & 7333 & $18.60 \%$ & $12: 09: 02$ & & & and peer2 failed \\
\hline
\end{tabular}

Table 4.3 Impact of Policy on TotalProcessingTime (3)

As shown in the Table 4.3, when the total routes injected increased to 39427 , the TestingNode cannot even apply policies on $18.60 \%$ of the total routes. This seems to confirm our hypothesis from previous experiments that the CPU is too busy (due to the 
linear search) and does not get to send out Keepalive messages before the HoldTimer expires. What if we increase the value of the HoldTimer and Keepalive timer? With this question, we conducted another two experiments, 4 and 5, as described below.

\subsubsection{Experiment 4}

Characteristics of the experiment:

- we announce 40451 routes (TotalRoutes) through BGPSim to the TestingNode.

- set simple AS import policies to reject $0.00 \%, 24.68 \%$ of the TotalRoute respectively.

- no export policy.

- Use default HoldTime $=90 \mathrm{msec}$, KeepAlive $=30 \mathrm{msec}$

The results are shown in Table 4.4:

\begin{tabular}{|c|c|c|c|c|c|c|c|}
\hline \#routes & Accepted & Rejected & $\%$ rejected & ISTART & STOP & DELAY & Comments \\
\hline 40,451 & 40,451 & p & $0.00 \%$ & $10: 34: 39$ & $10: 37: 56$ & $0: 03: 17$ & \\
\hline 40,451 & 30,466 & 9,985 & $24.68 \%$ & & & & $\begin{array}{l}\text { Connection to Peer1 } \\
\text { and Peer2 failed } \\
\text { HOLD TIME }=90 \\
\text { KEEPALIVE }=30\end{array}$ \\
\hline
\end{tabular}

Table 4.4 Impact of Policy on TotalProcessingTime (4)

The Hold Time is the maximum length of time that one endpoint will wait to hear something from the other endpoint (either an UPDATE message or a KEEPALIVE message) before assuming that the BGP session is down. A nonzero Hold Time must be 
at least three seconds. The Hold Time chosen for the BGP session is the minimum of the value configured locally and the value advertised by the neighbor. BGP protocol explicitly allows neighbors to reject an OPEN message if the value of the Hold Time field is not acceptable. [RFC1771]

\subsubsection{Experiment 5}

Characteristics of the experiment:

- we announce 40451 routes (TotalRoutes) through BGPSim to the TestingNode.

- set simple AS import policies to reject $24.68 \%$ of the TotalRoutes.

- no export policy.

- set HoldTime $=180 \mathrm{sec}$, KeepAlive $=60 \mathrm{sec}$

The results are shown in Table 4.5

\begin{tabular}{|c|c|c|c|c|c|c|c|}
\hline \#routes & Accepted & Rejected & $\%$ rejecte & START & STOP & DELAY & Comments \\
\hline 40,451 & 30,466 & 9,985 & $24.68 \%$ & $10: 45: 58$ & $10: 52: 17$ & 0:06:19 & $\begin{array}{l}\text { HOLD TIME }=180 \\
\text { KEEPALIVE }=60\end{array}$ \\
\hline
\end{tabular}

Table 4.5 Impact of Policy on TotalProcessingTime (5)

We can observe that, in case 2 of the experiment 4 , which had the same parameters as experiment 5 except that HoldTime and KeepAlive was two times shorter (HoldTime = $90 \mathrm{sec}$ and KeepAlive $=30 \mathrm{sec}$ ), the TestingNode was too busy at handling the incoming announcements and applying the import BGP policy, and didn't get to send out the 
KeepAlive message before the timeout. Therefore, Peerl thought that the other end was down, and dropped the BGP connection.

In experiment 5, we increased the value of HoldTime to $180 \mathrm{sec}$ and the value of KeepAlive to $60 \mathrm{sec}$, and we could observe that the TestingNode was able to handle 40451 announcements with applying the BGP import policy on $24.68 \%$ of them. Thus, from this experiment, we could conclude that the value of HoldTime and KeepAlive time is a factor in the router scalability. When these values are larger, the router is able to handle more routes announcements without failures. However, the side effect that larger values can result in a slow detection of the network topology changes. According to [RFC1771], the suggested value for Hold Time is 90 seconds, and the suggested value for KeepAlive is one third of the Hold Time, which is 30 seconds. However, these values may not be optimal for all cases, and more study is necessary for understanding how to choose them.

\subsubsection{Experiment 6}

Next we want to study the impact of the number of peers on the TotalProcessingTime by conducting the following experiment.

Characteristics of the experiment:

- we announce 40451 routes (TotalRoutes) through BGPSim to the TestingNode.

- set simple AS import policies to reject $24.68 \%$ of the TotalRoutes.

- no export policy.

- set to default value: HoldTime $=180 \mathrm{msec}$, KeepAlive $=30 \mathrm{msec}$

- No peer2 configured 
The results are shown in Table 4.6:

\begin{tabular}{|l|l|l|l|l|l|l|l|}
\hline \#routes & Accepted & Rejected & \%rejected & & & & \\
\hline 40,451 & 30,466 & 9,985 & $24.68 \%$ & $11: 10: 04$ & $11: 15: 20$ & $0: 05: 16$ & No Peer2 \\
\hline
\end{tabular}

Table 4.6 Impact of Policy on TotalProcessingTime (6)

In experiment 6 , when we brought down the peer session between the TestingNode and Peer2 before Peer1 started to send the advertisements, we found that the TestingNode was able to handle as many as 40451 new routes advertisement and applied import BGP policy on $24.68 \%$ of the total routes. So we could confirm that the number of peers is indeed a factor that impacts directly the routing convergence time. The more peers a router has, the longer it will take to process the routing updates.

\subsubsection{Observations from Experiments 1 to 6}

From this set of experiments, we can summarize our observations as the following:

- as the percentage of the routes to which routing polices are applied increases, the TotalProcessing Time increases almost linearly.

- as the number of routes learned increases, the TotalProcessingTime increases, but the maximum number of routes to which routing policies can be applied decreases. 
- the value of the HoldTime and KeepAlive is a factor of the router's scalability.

- the number of peers to propagate the updates to is also a factor that can directly impact the routing convergence time. The more peers a router has, the longer time it will take to process a given number of routing updates.

\subsection{Study of Effect of Route Stability on TotalProcessingTime}

\subsubsection{Experiments 7}

In this experiment, we use BGPSim to generate a total of 1000 routes and advertise them to TestingNode. To simulate the Internet Instability, we make $50,100,150,200,400$, 600,800 routes flapping respectively, and then we study the impact of route stability on the TotalProcessingTime.

As described in section 3.1, the network-list is used to simulate routing changes. We use stability to simulate the flapping of the routes. Routes are first announced and then withdrawn after a given time.

The results from the experiment are shown in Table 4.7 and Figure 4.4.

\begin{tabular}{|l|l|}
\hline \# of routes flapped & TotalProcessingTime (msec) \\
\hline 50 & 1564 \\
\hline 100 & 1787 \\
\hline 150 & 1920 \\
\hline 200 & 2252 \\
\hline
\end{tabular}




\begin{tabular}{|l|l|}
\hline 400 & 4120 \\
\hline 600 & 5794 \\
\hline 800 & 7236 \\
\hline 1000 & 7875 \\
\hline
\end{tabular}

Table 4.8 Impact of Instability on TotalProcessingTime

\section{Effect of Route Stablility on Processing Time}

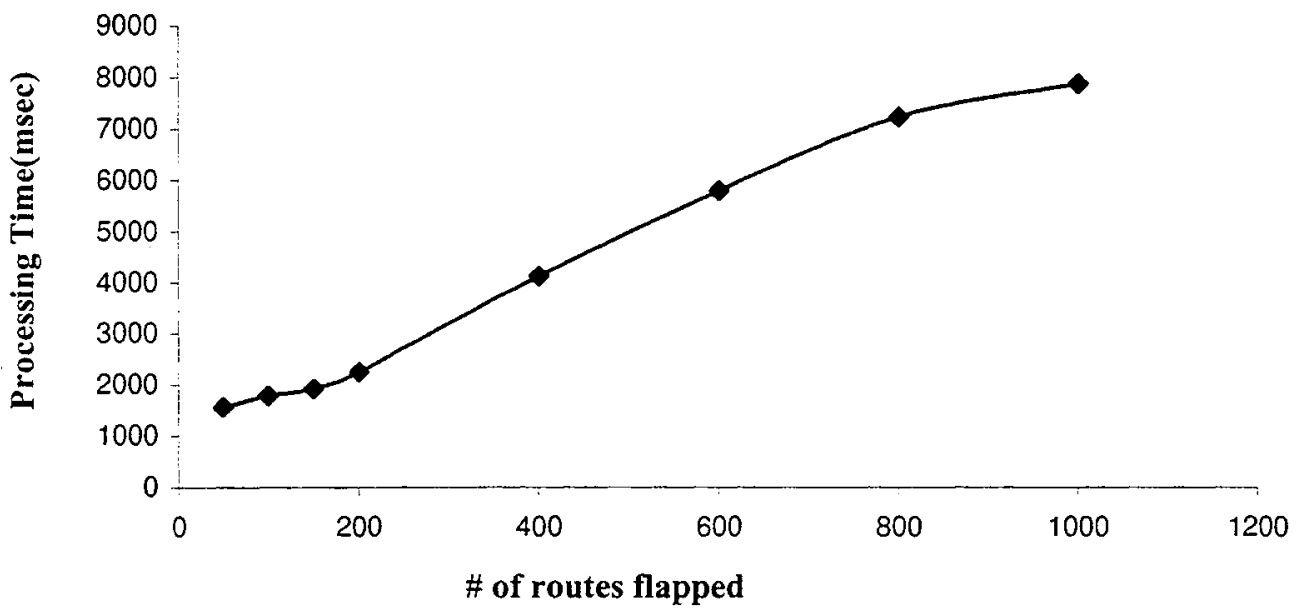

Figure 4.4 Effect of Route Stability on TotalProcessingTime

\subsubsection{Observations from Experiment 7}

As shown from the Figure 4.4, as the number of flapping routes increases, the TotalProcessingTime for the TestingNode to process the advertisement of the routes will increase. This means that the more unstable inter-domain routing is, the longer the router will take to complete the routing convergence. 


\subsection{Study of impact of number of routes advertised on TotalProcessingTime}

\subsubsection{Experiments 8}

In this experiment, we aim to study how the number of route advertisements will affect the routing convergence time. First, we bring down the peer session between TestingNode and Peer2. (see Figure 4.1) Then, we use BGPSim (Peer1) to simulate to advertise 10000 routes to TestingNode. After TestingNode completes processing all of the routes and becomes stable, we bring up the BGP peer session between TestingNode and Peer2. Then we use Quantify to measure the time period from the beginning of the first route till the end of advertising the last route.

The experimental results are shown in Table4.9 and Figure 4.5

\begin{tabular}{|l|l|}
\hline \# of routes advertised & TotalProcessingTime (msec) \\
\hline 10000 & 500 \\
\hline 20000 & 1215 \\
\hline 30000 & 1506 \\
\hline 40000 & 2163 \\
\hline 50000 & 2389 \\
\hline 60000 & 3465 \\
\hline
\end{tabular}

Table 4.9 Impact of \#Routes Advertised on TotalProcessingTime 


\section{Impact of \# of Routes Advertised}

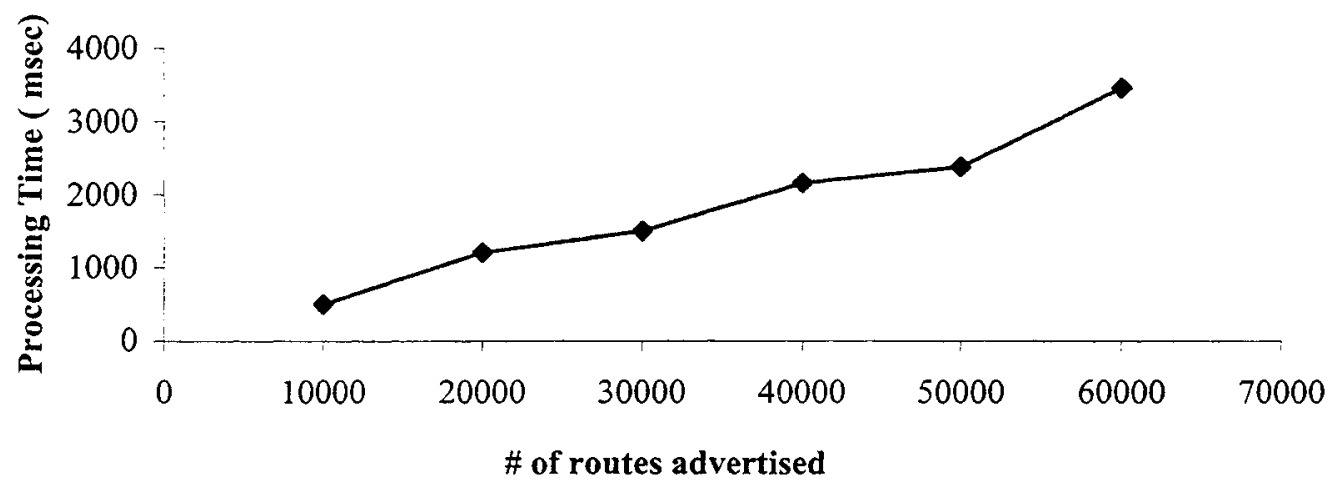

Figure 4.5 Impact of \#Routes Advertised on TotalProcessingTime

\subsubsection{Observations from Experiment 8}

From the result of this set of experiments shown in the Figure 4.5, the number of route advertisements affects the TotalProcessingTime. That is the more routes it advertises the longer the TotalProcessingTime is.

\subsection{Summary}

In this chapter, we described several sets of experiments to study the factors that have direct impact on the routing table update. We used the BGPSim tool to generate the BGP traffic for two BGP routers, and measured their performance with the Quantify tool. It is necessary to mention that when we looked at the implementation details, we found that a sorted linked list is a very commonly used data structure in this particular vendor's BGP implementation. A linear search algorithm is used to process the data stored in the linked 
list. We believe this is the reason why the value of the TotalProcessingTime increases almost linearly with the number of announcements, number of peers and number of routes to which a policy is applied. If a better search algorithm will be chosen (such as binary search, or hash) it will reduce the negative impact on the TotalProcessingTime. This could help to fasten the routing convergence and to improve the stability of the Internet. 


\section{CHAPTER 5. CONCLUSIONS}

This chapter summarizes the work done in the thesis and identifies directions for future work.

\subsection{Summary and Conclusions}

The contributions of the thesis work can be summarized as follows:

- Development of a Perl script to analyze the raw BGP traffic data. The script is able to process very large amounts of data, and to perform statistical analysis.

- Statistical analysis of the inter-domain BGP traffic, and its results.

- Experimental performance measurements of BGP routing table update, identifying factors that have an impact on the BGP routing convergence time. The measurements were performed under workload generated by BGPSim.

- Implementation of an Expect script to automatically generate the BGPSim configuration files necessary for conducting the measurements.

The result of our statistical analysis of raw BGP traffic data was compared with previous results published by other researchers in 1997 and 1999. We were able to observe general trends, and to summarize some facts that can be useful to future researchers who will analyze the characteristics of the Internet traffic in the next years. Here is a summary of our findings:

- From 1996 to 2001 , even though the size of the Internet has increased dramatically in that period, the average number of BGP updates per day has 
dropped by an order of magnitude (from 3-5 millions to 0.3 millions) at the MAEEAST exchange router. The significant drop is mainly due to improvements in the routing software and hardware provided by various vendors, and to some new technologies introduced, such as high aggregation, flapping damping, etc.

- In 2001, the mean number of BGP updates at Mae-East per month was 11,792,462, out of which 9,422,408 were Announcements and 2,370,054 Withdrawals.

- In 2001, the mean arrival rate of the BGP updates at Mae-East was 4.47/sec.

- From our study, we can still observe a high percentage of update redundancy. Over the 9 months study period, $56.19 \%$ of updates were found to be redundant. However, this is much better than $99 \%$ redundant updates reported in [Labovitz97].

- We defined two redundancy indicators that give the percentage of redundant Announcements and Withdrawals. Surprisingly, we found that in 2001 the DownRedundIndicator was 0 , which means that there were no redundant Withdrawal messages and all of the redundant updates were Announcements. This represents an important change from the results reported in [Labovitz97] where the majority of the redundant updates were Withdrawals. We found that in 2001 the mean UpRedundancyIndicator was 1.738 .

- The trend in terms of the number of the BGP routing information updates is continuously decreasing in 2001, which matches the trend from [Labovitz99].

- By introducing the concept of Network Livability, which shows the percentage of time when a network (prefix) is up over the prefix's lifetime within the study 
period, we can see that in 2001 , more than $80 \%$ of prefixes (networks) were up over $70 \%$ of the time. From this result, we can conclude that most prefixes are relatively stable, and the inter-domain routing instability stems mostly from a small percentage of prefixes. However, we are not sure if the cause is the originating networks itself or some unstable nodes along the route.

- BGP traffic does show weekly and daily patterns. The magnitude of BGP traffic exhibits the same significant weekly, daily patterns as the network usage and congestion, which is also stated in [Labovitz99]. However, we give more data to support this conclusion.

- The majority of routes are relatively stable, and only a small percentage of the routes are contributing the most to the instability of inter-domain routing.

- We used k-means clustering analysis technique to analyze the stability of the routes and provide guidance for choosing the BGPSim parameters that represent the network stability.

- We observed that an aggregate prefix length from 16 to 24 bits is commonly used in inter-domain routing, and the length of 24 is the most often used. This can give us an idea what are the sizes of the prefixes in the real world Internet.

From the measurements conducted on a given BGP router, it was confirmed that several factors have an impact on the scalability of the router, such as the number of routes learned, number of routes advertised, number of routes to apply policy on, number of peers, the value of the HoldTimer and Keepalive, number of routes flapping etc. We also observed a common problem that appears in some router implementations: the data 
structures and algorithms used are not always the most efficient. For example, we believe that by using better search algorithms in the BGP router implementation we have studied, the negative impact of various factors on the TotalProcessingTime can be reduced, and thus the router can be made more scalable.

\subsection{Future work}

In our research, we tried to characterize some properties of the BGP traffic, but have not considered all the factors. Specifically, we did not study the characteristics of the following attributes: AS-PATH, LOCAL_PREF, COMMUNITY, MED, etc, which are shown in the BGP traces. This could be a subject for future work.

Also, during our research, we observed that there still are pathologic routing behaviors in today's BGP traffic, such as forwarding loops and redundant updates. Those anomalies

may be caused by some misconfigurations or improper routing policies, but more investigation is necessary to understand in-depth their root causes. In general, more research is necessary to understand and correct all the causes for Internet instability. 


\section{REFERENCES}

[Aldenderfer84] Aldenderfer, Mark S. "Cluster Analysis”, Sage Publications, 1984.

[Chinoy93]

Bilal Chinoy, "Dynamics of Internet Routing Information", Proc. of SIGCOMM'93, 1993.

[Cisco]

Cisco, "BGP4 Case Studies/Tutorial", http://www.cisco.com

[Cowie01]

James Cowie, Andy Ogielski, “ Global Routing Instabilities during Code Red II and Nimda Worm Propagation Preliminary Report", http://www.renesys.com/projects/bgp instability, 2001.

[Ferguson98] Paul Ferguson, "BGP Intro Tutorial", 1998.

http://www.academ.com/nanog/

[Farrow02] Rik Farrow, "Routing Instability on the Internet”, Network Magazine, 2002.

[Griffin99] T.G. Griffin, Gordon Eilfong, "An Analysis of BGP Convergence Properties", Procedings of SIGCOMM'99, 1999.

[Govindan97] Ramesh Govindan, Anoop Reddy, "An Analysis of Internet InterDomain Topology and Route Stability", Proceedings of IEEE INFOCOM'97, 1997.

[IPMA] Internet Performance Measurement and Analysis project (IPMA), http://www.merit.edu/ipma.

[Jain91]

[Labovitz97] Craig Labovitz, G.Robert Malan, and Farnam Jahanian, "Internet Routing Instability", Proceedings of ACM SIGCOMM' 97, 1997.

[Labovitz99] Craig Labovitz, G.Robert Malan, and Farnam Jahanian, “Origins of Internet Routing Instability", Proceedings of INFOCOM'99, New York, NY, June, 1999.

[Labovitz99-a] Craig Labovitz, Masaki Hirabaru, "Routing Technology Final Report", http://www.merit.edu/ labovit/, 1999. 
[Labovitz00-a] Craig Labovitz and Abha Ahuja, "Experimental Study of Internet Stability and Wide-Area Backbone Failures", Proceedings of IEEE INFOCOM '00, 2000.

[Labovitz00-b] Craig Labovitz, "An Experimental Study of Internet Routing Convergence", Proceedings of SIGCOMM'00, 2000.

[Labovitz01] Craig Labovitz, Ahba Ahuja, "The Impact of Internet Policy and Topology on Delayed Routing Convergence", Proceedings of IEEE INFOCOM '01, 2001.

[Malan98] G.Robert Malan and Farnam Jahanian, "An Extensible Probe Architecture for Network Protocol Performance Measurement", Proceedings of ACM SIGCOMM' 98, 1998.

[Mahajan98] Mahajan Dvaid Wetherall " Understanding BGP misconfiguration", Proceedings of SIGCOMM'02, 2002,

[Menasce99] Daiel A. Menasce, Virgilio A.F. Almeida, "A Methodology for Workload Characterization of E-commerce sites", E-COMMERCE 99, 1999, http://www.informatik.uni-trier.de/ ley/db/indices/atree/a/Almeida:Virgilio.html

[Merit99] Merit Networks, "Multithreaded Routing Toolkit", http://www.mrt.net, 1999

[Paxson96] V.Paxson, "End-to-end Routing Behaviors in the Internet", Proceedings of SIGCOMM'96, 1996.

[RFC1519] V.Fuller, T.Li, J.Yu, and K.Varadhan. Classless Inter-Domain Routing(CIDR): An Address Assignment and Aggregation Strategy. RFC1519, 1993.

[RFC1771] Rekhter, Yakov, and Tony Li "Border Gateway Protocol 4", RFC1771, 1995

[RFC1966] T-bates, R. Chandra "BGP Route Reflection An alternative to full mesh”, RFC1966, 1996

[RFC1998] E. Chen, T. Bates "An Application of the BGP Community Attribute in Multi-home Routing" RFC1998, 1998.

[RFC2439] C. Villamizar, R. Chandra "BGP Route Flap Damping", RFC2439, 1998.

[Shaikh00] Aman Shaikh, Lampros Kalampoukas, Rohit Dube, Anujan Varma "Routing Stability in Congested Networks: Experimentation and Analysis", Proceedings of SIGCOMM'00, 2000, 
[Sisu]

[Stewart99]

[Weing97] http://www.engr.sjsu.edu/ knapp/HCIRDFSC/C/k_means.htm

John Stewart III, "BGP-4: Interdomain Routing in the Internet", Addison Wesley, 1999.

Tim Weingarten "Internet Backbones and Router Instability", Proceedings of TCOM'97, 1997. 